\title{
LUIGI AMBROSIO
}

GIANLUCA CRIPPA

\section{STEFANIA MANIGLIA}

\section{Traces and fine properties of a $B D$ class of vector fields and applications}

Annales de la faculté des sciences de Toulouse $\sigma^{e}$ série, tome 14, $\mathrm{n}^{\mathrm{o}} 4$ (2005), p. 527-561

<http://www.numdam.org/item?id=AFST_2005_6_14_4_527_0>

(C) Université Paul Sabatier, 2005, tous droits réservés.

L'accès aux archives de la revue «Annales de la faculté des sciences de Toulouse » (http://picard.ups-tlse.fr/ annales/) implique l'accord avec les conditions générales d'utilisation (http://www.numdam.org/conditions). Toute utilisation commerciale ou impression systématique est constitutive d'une infraction pénale. Toute copie ou impression de ce fichier doit contenir la présente mention de copyright.

\section{NumDam}

Article numérisé dans le cadre du programme Numérisation de documents anciens mathématiques http://www.numdam.org/ 


\title{
Traces and fine properties of a $B D$ class of vector fields and applications ${ }^{(*)}$
}

\author{
Luigi Ambrosio (1), Gianluca Crippa ${ }^{(1)}$, \\ Stefania Maniglia ${ }^{(2)}$
}

\begin{abstract}
In this paper we study the fine properties and the trace properties of a class of vector fields of the form $C=w B$, where $w$ is a locally bounded scalar function and $B$ is locally bounded and with finite deformation. Assuming also that the distributional divergence of $C$ is a locally finite measure, we relate the (distributional) trace of $C$ on hypersurfaces to the pointwise behaviour of $w$. We study also the behaviour of these traces under the transformation $w B \longmapsto h(w) B$, with $h \in C^{1}$, proving a chain rule for traces.

As a consequence of these results we show that DiPerna-Lions theory can be extended to special vector fields with bounded deformation. In the case when $B$ is locally $B V$ we obtain also estimates on the size of the approximation discontinuity and approximate jump sets of $w$.
\end{abstract}

RÉSUMÉ. - Dans cet article, nous étudions les propriétés fines et les propriétés de trace pour une classe de champs de vecteurs de la forme $C=w B$, où $w$ est une fonction scalaire bornée et $B$ est localement bornée et avec déformation bornée. Dans l'hypothèse que la divergence au sens de la distribution de $C$ sur une hypersurface, avec le comportement ponctuel de $w$. Nous étudions aussi le comportement de ces traces par rapport à la transformation $w B \longmapsto h(w) B$, avec $h \in C^{1}$, et nous démontrons une formule explicite pour les traces. Comme conséquence de ces résultats nous démontrons que la théorie de DiPerna-Lions peut s'étendre aux champs de vecteurs spéciaux avec une déformation bornée. Lorsque $B$ est localement $B V$ nous obtenons aussi les estimations sur la taille des ensembles de «discontinuité » approximative de $w$.

(*) Reçu le 19 mars 2004, accepté le 4 avril 2005

This article was written at the occasion of the Fermat Prize 2003 awarded to L. Ambrosio for his impressive contributions to the calculus of variations and geometric measure theory, and their link with partial differential equations.

(1) Scuola Normale Superiore, Piazza dei Cavalieri 7, 56126 Pisa (Italy).

E-mail: l.ambrosio@sns.it, g.crippa@sns.it

(2) Dipartimento di Matematica, Via Buonarroti, 56100 Pisa (Italy).

E-mail: maniglia@mail.dm.unipi.it 


\section{Introduction}

This paper is devoted to the study of the trace properties and of the pointwise behaviour of vector fields $C$ in $\mathbb{R}^{d}$ of the form $w B$, where $w$ is a scalar function, the distributional divergence $D \cdot C$ of $C$ is a Radon measure and $B$ is a weakly differentiable vector field. We will consider in particular the case when $B$ has locally bounded deformation (and we write $B \in B D_{\text {loc }}$ ), i.e. the symmetric distributional gradient $E B$ of $B$ is a vector-valued Radon measure (see [28], [5]). This regularity class seems the natural one in view of the following facts: on one hand the first author established in [3] an extension of DiPerna-Lions theory [21] to the case when $B$ has bounded variation; on the other hand it was proved in [16] that still the theory works under the assumption that $E B \in L_{\mathrm{loc}}^{1}$. So a natural attempt is to improve both results extending the theory to the case when $E B$ is only a Radon measure. We don't achieve completely this goal, but we obtain partial results and some auxiliary facts of general interest, that will be used in the forthcoming paper [7].

The plan of the paper is the following. In $\S 2$ we fix our main notation and recall the main facts about functions of bounded variation and functions of bounded deformation. In particular we show in Proposition 2.5 that the splitting of the difference quotients of a $B V$ function into a strongly converging part and a weakly converging part (one of the main tools used in [3] to show that distributional solutions are indeed renormalized solutions) extends to $B D$ functions, taking the symmetric difference quotients into account. This leads to the fact, proved in Theorem 2.6, that all limit points of the modulus of the commutators

$$
(D \cdot(w B)) * \rho_{\epsilon}-D \cdot\left(\left(w * \rho_{\epsilon}\right) B\right)
$$

as $\epsilon \downarrow 0$ are singular with respect to the Lebesgue measure $\mathcal{L}^{d}$, provided the convolution kernel is radial.

In $\S 3$ we study the trace properties of locally bounded vector fields whose divergence is a measure. Almost all the results of this section appeared in [17] (see also [9], where first the existence of the normal trace was proved, the unpublished paper [11] that influenced a lot our work and the more recent paper [18], where even measure fields are considered), but we prefer to write all results in a self-contained way, consistent with our purposes. The starting point is that the normal trace $\operatorname{Tr}(C, \partial \Omega)$ on a bounded open set $\Omega$ with a $C^{1}$ boundary can be defined as a distribution, by the identity

$$
\langle\operatorname{Tr}(C, \partial \Omega), \varphi\rangle:=\int_{\Omega} \nabla \varphi \cdot C d x+\int_{\Omega} \varphi d D \cdot C \quad \forall \varphi \in C_{c}^{\infty}\left(\mathbb{R}^{d}\right) .
$$


It turns out that this distribution is induced by a locally bounded function defined on $\partial \Omega$, which coincides with the pointwise normal component of $C$ on $\partial \Omega$ for "generic" open sets $\Omega$ (see Proposition 3.6 for a precise statement). Moreover the trace operator is local not only on open sets, but also in the following stronger sense: for any pair of $C^{1}$ open sets $\Omega_{1}, \Omega_{2}$ we have

$\operatorname{Tr}\left(C, \partial \Omega_{1}\right)=\operatorname{Tr}\left(C, \partial \Omega_{2}\right) \quad \mathcal{H}^{d-1}$-a.e. on $\left\{x \in \partial \Omega_{1} \cap \partial \Omega_{2}: \nu_{\Omega_{1}}(x)=\nu_{\Omega_{2}}(x)\right\}$, where $\nu_{\Omega_{1}}$ and $\nu_{\Omega_{2}}$ are the outer normals to $\Omega_{1}$ and $\Omega_{2}$ respectively (no regularity is imposed on the intersection of the two boundaries). This fact is crucial in order to extend the trace operator to countably $\mathcal{H}^{d-1}$-rectifiable sets which, in general, are not locally the boundary of an open set.

In $\S 4$ we go back to our special class of vector fields $C=w B$ with $w, B \in L_{\mathrm{loc}}^{\infty}$ and $B \in B D_{\mathrm{loc}}$ and we establish the chain rule for traces

$$
\operatorname{Tr}(h(w) B, \partial \Omega)=\operatorname{Tr}(B, \partial \Omega) h\left(\frac{\operatorname{Tr}(w B, \partial \Omega)}{\operatorname{Tr}(B, \partial \Omega)}\right) \quad \forall h \in C^{1}(\mathbb{R}) .
$$

Its proof requires the quantitative version of the commutator estimate given in $\S 2$ and a suitable extension argument, based on Gagliardo's theorem.

In $\S 5$ we show how the DiPerna-Lions theory can be extended to special vector fields of bounded deformation, i.e. those fields $B \in B D_{\text {loc }}$ such that the singular part of $E B$ is concentrated on an $\mathcal{H}^{d-1}$-rectifiable set. We have also to assume, as in [3], that the distributional divergence of $B$ is absolutely continuous with respect to $\mathcal{L}^{d}$. The key property is the renormalization lemma

$$
B \cdot \nabla w=c \mathcal{L}^{d} \quad \Longrightarrow \quad B \cdot \nabla(h(w))=c h^{\prime}(w) \mathcal{L}^{d} \quad \text { for any } h \in C^{1}(\mathbb{R}) .
$$

Unlike [3] (see also [25], [12], [20]) its proof can not be achieved by choosing very anisotropic convolution kernels, as for $B D$ functions only radial kernels ensure good estimates of the commutator. We use instead the chain rule for traces to rule out the possibility of a concentration of $B \cdot \nabla(h(w))$ on hypersurfaces. In the last part of the section we illustrate several standard consequences of the renormalization lemma: well-posedness of the continuity equation, existence and uniqueness of regular Lagrangian flows, and stability of regular Lagrangian flows with respect to smooth approximations.

Finally in $\S 6$ we analyze the pointwise behaviour of $w$. This is an important issue in the perspective [7] of defining a "precise representative" of $w$ to be used in a kind of chain-rule formula for the computation of $D \cdot(h(w) B)$ even when $D \cdot B$ is not absolutely continuous with respect to $\mathcal{L}^{d}$, thus extending all renormalization lemmas known so far. We show first 
the existence of approximate one-sided limits on $C^{1}$ hypersurfaces, relating them to the distributional trace, and then, using the coarea formula, we are able to prove when $B \in B V_{\text {loc }}$ the existence of the one-sided approximate limits $|D B|$-a.e. However, we are able to show these properties only on the set where the vector field is transversal, in a suitable sense, to its derivative (see (6.5), (6.6)).

\section{Main notation and preliminary results}

We denote by $\mathcal{L}^{d}$ the Lebesgue measure in $\mathbb{R}^{d}$ and by $\mathcal{H}^{k}(E)$ the Hausdorff $k$-dimensional measure of a set $E \subset \mathbb{R}^{d}$. In the sequel we denote by $\Omega$ a generic open set in $\mathbb{R}^{d}$. Given a nonnegative Borel measure $\mu$ in $\Omega$ we say that $\mu$ is concentrated on a Borel set $F$ if $\mu(\Omega \backslash F)=0$. For a Borel set $F \subset \Omega$, the restriction $\mu\llcorner F$ is defined by

$$
\mu\llcorner F(E):=\mu(F \cap E) \quad \text { for any Borel set } E \subset \Omega .
$$

The same operation can be defined for vector valued measures $\mu$ with finite total variation in $\Omega$. We will sometimes use the following differentiation property (see for instance [23] or Theorem 2.56 of [8]):

$$
\mu\left\llcorner E=0 \quad \Longrightarrow \quad \mu\left(B_{r}(x)\right)=o\left(r^{k}\right) \text { for } \mathcal{H}^{k} \text {-a.e. } x \in E .\right.
$$

The approximate discontinuity set $S_{B} \subset \Omega$ of a locally summable $B$ : $\Omega \rightarrow \mathbb{R}^{m}$ and the approximate limit are defined as follows: $x \notin S_{B}$ if and only if there exists $z \in \mathbb{R}^{m}$ satisfying

$$
\lim _{r \downarrow 0} r^{-d} \int_{B_{r}(x)}|B(y)-z| d y=0 .
$$

The vector $z$, if exists, is unique and denoted by $\tilde{B}(x)$, the approximate limit of $B$ at $x$. It is easy to check that the set $S_{B}$ is Borel and that $\tilde{B}$ is a Borel function in its domain (see $\S 3.6$ of [8] for details). By Lebesgue differentiation theorem the set $S_{B}$ is Lebesgue negligible and $\tilde{B}=B \mathcal{L}^{d}$-a.e. in $\Omega \backslash S_{B}$.

In a similar way one can define the approximate jump set $J_{B} \subset S_{B}$, by requiring the existence of $a, b \in \mathbb{R}^{m}$ with $a \neq b$ and of a unit vector $\nu$ such that

$$
\lim _{r \downarrow 0} r^{-d} \int_{B_{r}^{+}(x, \nu)}|B(y)-a| d y=0, \quad \lim _{r \downarrow 0} r^{-d} \int_{B_{r}^{-}(x, \nu)}|B(y)-b| d y=0,
$$

where

$$
\begin{gathered}
\left\{\begin{array}{c}
B_{r}^{+}(x, \nu):=\left\{y \in B_{r}(x):\langle y-x, \nu\rangle>0\right\} \\
B_{r}^{-}(x, \nu):=\left\{y \in B_{r}(x):\langle y-x, \nu\rangle<0\right\}
\end{array}\right. \\
-530-
\end{gathered}
$$


The triplet $(a, b, \nu)$, if exists, is unique up to a permutation of $a$ and $b$ and a change of sign of $\nu$, and denoted by $\left(B^{+}(x), B^{-}(x), \nu(x)\right)$, where $B^{ \pm}(x)$ are called approximate one-sided limits of $B$ at $x$. It is easy to check that the set $J_{B}$ is Borel and that $B^{ \pm}$and $\nu$ can be chosen to be Borel functions in their domain (see again $\S 3.6$ of [8] for details).

For $B \in L_{\text {loc }}^{1}\left(\Omega ; \mathbb{R}^{m}\right)$ we denote by $D B=\left(D_{i} B^{j}\right)$ the derivative in the sense of distributions of $B$, i.e. the $\mathbb{R}^{m \times d}$-valued distribution defined by

$$
D_{i} B^{j}(\varphi):=-\int_{\Omega} B^{j} \frac{\partial \varphi}{\partial x_{i}} d x \quad \forall \varphi \in C_{c}^{\infty}(\Omega), \quad 1 \leqslant i \leqslant d, 1 \leqslant j \leqslant m .
$$

In the case when $m=d$ we denote by $E B$ the symmetric part of the distributional derivative of $B$, i.e.,

$$
E B:=\left(E_{i j} B\right), \quad E_{i j} B:=\frac{1}{2}\left(D_{i} B^{j}+D_{j} B^{i}\right) \quad 1 \leqslant i, j \leqslant d .
$$

Definition 2.1 ( $B V$ AND $B D$ FUnCtions). - We say that $B \in L^{1}\left(\Omega ; \mathbb{R}^{m}\right)$ has bounded variation in $\Omega$, and we write $B \in B V\left(\Omega ; \mathbb{R}^{m}\right)$, if $D B$ is representable by an $\mathbb{R}^{m \times d}$-valued measure, still denoted with $D B$, with finite total variation in $\Omega$.

We say that $B \in L^{1}\left(\Omega ; \mathbb{R}^{d}\right)$ has bounded deformation in $\Omega$, and we write $B \in B D(\Omega)$, if $E_{i j} B$ is a Radon measure with finite total variation in $\Omega$ for any $i, j=1, \ldots, d$.

We consider, for $B \in B V_{\text {loc }}\left(\Omega ; \mathbb{R}^{m}\right)$, the canonical Radon-Nikodým decomposition of $D B$ into an absolutely continuous part $D^{a} B$ with respect to $\mathcal{L}^{d}$ and a singular part $D^{s} B$ with respect to $\mathcal{L}^{d}$. Analogously, for $B \in B D_{\text {loc }}(\Omega)$, we consider the Radon-Nikodým decomposition of $E B$ into an absolutely continuous part $E^{a} B$ with respect to $\mathcal{L}^{d}$ and a singular part $E^{s} B$ with respect to $\mathcal{L}^{d}$. We denote also by $\mathcal{E} B$ the Borel map with values into symmetric $d \times d$ matrices representing the density of $E^{a} B$ with respect to $\mathcal{L}^{d}$, i.e. $E^{a} B=\mathcal{E} B \mathcal{L}^{d}$.

The distributional divergence $D \cdot B:=\sum_{i} D_{i} B^{i}=\sum_{i} E_{i i} B$ is a well defined measure with finite total variation in $\Omega$ when $B \in B D(\Omega)$; defining $\operatorname{div} B$ as the trace of $\mathcal{E} B$, the splitting of $D \cdot B$ into absolutely continuous and singular part with respect to $\mathcal{L}^{d}$ can be read as follows:

$$
D \cdot B=\operatorname{div} B \mathcal{L}^{d}+\sum_{i=1}^{d} E_{i i}^{s} B=\operatorname{div} B \mathcal{L}^{d}+D^{s} \cdot B .
$$


Definition 2.2 (Countably $\mathcal{H}^{d-1}$-ReCtifiable SETs). - We say that $E \subset \mathbb{R}^{d}$ is a countably $\mathcal{H}^{d-1}$-rectifiable set if there exist (at most) countably many $C^{1}$ embedded hypersurfaces $\Gamma_{i} \subset \mathbb{R}^{d}$ such that

$$
\mathcal{H}^{d-1}\left(E \backslash \bigcup_{i} \Gamma_{i}\right)=0 .
$$

In a similar way, choosing oriented hypersurfaces $\Gamma_{i}$, one can define an orientation $\nu_{E}$ choosing pairwise disjoint Borel sets $E_{i} \subset \Gamma_{i}$ such that the union of the $E_{i}$ 's covers $\mathcal{H}^{d-1}$-almost all of $E$ and defining

$$
\nu_{E}:=\nu_{\Gamma_{\imath}} \quad \text { on } E_{i} .
$$

This orientation depends clearly on the choice of the decomposition, but only up to the sign, due to the fact that for any pair of $C^{1}$ hypersurfaces $\Gamma$ and $\Gamma^{\prime}$ we have $\nu_{\Gamma^{\prime}} \in\left\{-\nu_{\Gamma}, \nu_{\Gamma}\right\} \mathcal{H}^{d-1}$-a.e. on $\Gamma \cap \Gamma^{\prime}$.

We recall that for a $B V$ function $B$ the approximate discontinuity set $S_{B}$ and the jump set $J_{B}$ are countably $\mathcal{H}^{d-1}$-rectifiable and

$$
\mathcal{H}^{d-1}\left(S_{B} \backslash J_{B}\right)=0
$$

(see Theorem 4.5.9 in [23] or Theorem 3.78 in [8]). For functions $B \in B D(\Omega)$ it is known that $J_{B}$ is countably $\mathcal{H}^{d-1}$-rectifiable (see [5]) but the validity of (2.3) is still an open problem.

DEFINITION 2.3 ( $S B V$ AND $S B D$ FUnCTIONS). - We say that $B \in B V\left(\Omega ; \mathbb{R}^{m}\right)$ is a special function of bounded variation, and we write $B \in S B V\left(\Omega ; \mathbb{R}^{m}\right)$, if $D^{s} B$ is concentrated on a countably $\mathcal{H}^{d-1}$-rectifiable set. Analogously, we say that $B \in B D(\Omega)$ is a special function with bounded deformation, and we write $B \in S B D(\Omega)$, if $E^{s} B$ is concentrated on a countably $\mathcal{H}^{d-1}$-rectifiable set.

Since (see for instance Theorem 3.77 of [8])

$$
D B\left\llcorner F=\left(B^{+}-B^{-}\right) \otimes \nu_{B} \mathcal{H}^{d-1}\left\llcorner F \cap J_{B}\right.\right.
$$

for any countably $\mathcal{H}^{d-1}$-rectifiable Borel set $F \subset \Omega$, it turns out that $B \in S B V(\Omega)$ if and only if $\left|D^{s} B\right|$ is concentrated on $J_{B}$, and in some sense $J_{B}$ is the minimal set where the measure is concentrated. Analogous remarks hold in $B D(\Omega)$, due to the fact that (see Chapter II in [28])

$$
E B\left\llcorner F=\left(B^{+}-B^{-}\right) \odot \nu_{B} \mathcal{H}^{d-1}\left\llcorner F \cap J_{B}\right.\right.
$$


for any countably $\mathcal{H}^{d-1}$-rectifiable Borel set $F \subset \Omega$ (here $a \odot b$ denotes the symmetric tensor product of $a$ and $b$, i.e. $(a \otimes b+b \otimes a) / 2)$.

Let us recall now some fine properties of functions with bounded deformation that will be used in this paper, referring to [5] for detailed proofs and more informations. It is well known that, in analogy to what happens for $B V$ (see Section 3.11 of [8] for the corresponding statements in $B V$ ), the space $B D(\Omega)$ can be characterized by means of the one dimensional sections: a function $B \in L^{1}\left(\Omega ; \mathbb{R}^{d}\right)$ belongs to $B D(\Omega)$ if and only if, for every direction $\xi \in S^{d-1}:=\left\{\zeta \in \mathbb{R}^{d}:|\zeta|=1\right\}$, we have $B_{y}^{\xi} \in B V\left(\Omega_{y}^{\xi} ; \mathbb{R}\right)$ for $\mathcal{H}^{d-1}$ - a.e. $y \in \Omega^{\xi}$ and

$$
\int_{\Omega^{\xi}}\left|D B_{y}^{\xi}\right|\left(\Omega_{y}^{\xi}\right) d \mathcal{H}^{d-1}(y)<+\infty
$$

where $\Omega_{y}^{\xi}:=\{t \in \mathbb{R}: y+t \xi \in \Omega\}$ is the one dimensional section of $\Omega$ on the straight line passing through $y$ in the direction $\xi, \Omega^{\xi}:=\left\{y \in \pi_{\xi}: \Omega_{y}^{\xi} \neq \emptyset\right\}$ denotes the orthogonal projection of $\Omega$ onto $\pi_{\xi}$, the hyperplane orthogonal to $\xi$ passing through the origin, and $B_{y}^{\xi}:=B(y+t \xi) \cdot \xi$ for every $t \in \Omega_{y}^{\xi}$. i.e.

Furthermore, Fubini's theorem gives that $\int_{\Omega^{\xi}} D B_{y}^{\xi} d \mathcal{H}^{d-1}(y)=\langle E B \xi, \xi\rangle$,

$$
\begin{aligned}
\int_{\Omega^{\xi}}\left\langle D B_{y}^{\xi}, \varphi_{\xi, y}(\cdot)\right\rangle d \mathcal{H}^{d-1}(y) & =-\int_{\Omega^{\xi}}\left\langle B_{y}^{\xi}, \varphi_{\xi, y}^{\prime}\right\rangle d \mathcal{H}^{d-1}(y) \\
& =-\int_{\Omega} B \cdot \xi \frac{\partial \varphi}{\partial \xi} d x \\
& =\int_{\Omega} \varphi d\langle E B \xi, \xi\rangle \quad \forall \varphi \in C_{c}^{\infty}(\Omega),
\end{aligned}
$$

where $\varphi_{\xi, y}(t)=\varphi(y+t \xi)$. The structure theorem for $B D$ functions (see Theorem 4.5 of [5]) states that also the scalar products $\left\langle E^{a} B \xi, \xi\right\rangle$ and $\left\langle E^{s} B \xi, \xi\right\rangle$ can be recovered in an analogous way from the corresponding parts of the derivative of $B_{y}^{\xi}$, i.e.

$$
\left\langle E^{\sigma} B \xi, \xi\right\rangle=\int_{\Omega^{\xi}} D^{\sigma} B_{y}^{\xi} d \mathcal{H}^{d-1}(y) \quad \sigma=a, s
$$

and also that

$$
\left|\left\langle E^{\sigma} B \xi, \xi\right\rangle\right|=\int_{\Omega^{\xi}}\left|D^{\sigma} B_{y}^{\xi}\right| d \mathcal{H}^{d-1}(y) \quad \sigma=a, s .
$$

We also recall that for $B D$ functions the following uniform estimate of symmetric difference quotients (i.e. in the direction $z$ and with the scalar product along $z$ ) holds. 
LEMmA 2.4. - If $B \in B D_{l o c}(\Omega)$, then for any vector $z \in \mathbb{R}^{d}$ and any compact set $K \subset \Omega$ we have

$$
\int_{K}|(B(x+z)-B(x)) \cdot z| d x \leqslant|\langle E B z, z\rangle|\left(K_{|z|}\right)
$$

where $K_{|z|}$ is the open $|z|-$ neighbourhood of $K$, provided $|z|<\operatorname{dist}(K, \partial \Omega)$.

Proof. - Let $f \in C^{\infty}\left(\Omega ; \mathbb{R}^{d}\right)$ and let $z \in \mathbb{R}^{d}$, then for any compact set $K \subset \Omega$ we have

$$
\begin{aligned}
\int_{K}|(f(x+z)-f(x)) \cdot z| d x & =\int_{K}\left|\int_{0}^{1}\langle\nabla f(x+t z) z, z\rangle d t\right| d x \\
& \leqslant \int_{0}^{1} \int_{K}|\langle\nabla f(x+t z) z, z\rangle| d x d t \\
& \leqslant \int_{K_{|z|}}|\langle\nabla f(y) z, z\rangle| d y .
\end{aligned}
$$

So (2.6) is true for functions $f$ in $C^{\infty}\left(\Omega ; \mathbb{R}^{d}\right)$. Given a convolution kernel $\rho: \mathbb{R}^{d} \rightarrow[0,+\infty)$, let us define $B_{\epsilon}:=B * \rho_{\epsilon}$ and let us apply (2.6) to $B_{\epsilon}$ with $z^{\prime}=(1-\delta) z$ to get

$$
\int_{K}\left|\left(B_{\epsilon}\left(x+z^{\prime}\right)-B_{\epsilon}(x)\right) \cdot z^{\prime}\right| d x \leqslant\left|\left\langle E B_{\epsilon} z^{\prime}, z^{\prime}\right\rangle\right|\left(K_{\left|z^{\prime}\right|}\right) .
$$

Using Fatou's lemma and Jensen's inequality (see for instance Theorem 2.2(b) of [8]) we obtain

$$
\begin{aligned}
\int_{K}\left|\left(B\left(x+z^{\prime}\right)-B(x)\right) \cdot z^{\prime}\right| d x & \leqslant \liminf _{\epsilon \downarrow 0} \int_{K}\left|\left(B_{\epsilon}\left(x+z^{\prime}\right)-B_{\epsilon}(x)\right) \cdot z^{\prime}\right| d x \\
& \leqslant \limsup _{\epsilon \downarrow 0}\left|\left\langle E B_{\epsilon} z^{\prime}, z^{\prime}\right\rangle\right|\left(K_{\left|z^{\prime}\right|}\right) \\
& =\limsup _{\epsilon \downarrow 0}\left|\left\langle E B z^{\prime}, z^{\prime}\right\rangle * \rho_{\epsilon}\right|\left(K_{\left|z^{\prime}\right|}\right) \\
& \leqslant \limsup _{\epsilon \downarrow 0}\left|\left\langle E B z^{\prime}, z^{\prime}\right\rangle\right|\left(K_{\left|z^{\prime}\right|+\epsilon}\right) \\
& \leqslant\left|\left\langle E B z^{\prime}, z^{\prime}\right\rangle\right|\left(\overline{K_{\mid z^{\prime}} \mid}\right) .
\end{aligned}
$$

Recall that $z^{\prime}=(1-\delta) z$, so passing to the limit as $\delta \downarrow 0$ in the inequality

$$
\int_{K}\left|\left(B\left(x+z^{\prime}\right)-B(x)\right) \cdot z^{\prime}\right| d x \leqslant\left|\left\langle E B z^{\prime}, z^{\prime}\right\rangle\right|\left(K_{|z|}\right)
$$

and taking into account the strong continuity in $L_{\mathrm{loc}}^{1}$ of translations the thesis is achieved. 
We know that some properties of $B V$ functions can be suitably extended to $B D$ functions. For example the following proposition provides more information on the behaviour of the symmetric difference quotients of $B D$ functions and, as expected, we get a result similar to the one for difference quotients of $B V$ functions (see Theorem 2.4 of [3]).

Proposition 2.5. - Let $B \in B D_{\text {loc }}\left(\mathbb{R}^{d}\right)$ and let $z \in \mathbb{R}^{d} \backslash\{0\}$. Then the symmetric difference quotients

$$
\frac{B(x+\delta z)-B(x)}{\delta} \cdot \frac{z}{|z|}
$$

can be canonically written as $B_{\delta}^{1}(z)(x)+B_{\delta}^{2}(z)(x)$, where

$$
B_{\delta}^{1}(z)(\cdot) \longrightarrow\left\langle\mathcal{E} B(\cdot) z, \frac{z}{|z|}\right\rangle \quad \text { strongly in } L_{\mathrm{loc}}^{1}\left(\mathbb{R}^{d} ; \mathbb{R}\right) \text { as } \delta \downarrow 0
$$

and

$$
\limsup _{\delta \downarrow 0} \int_{K}\left|B_{\delta}^{2}(z)\right| d x \leqslant \frac{1}{|z|}\left|\left\langle E^{s} B z, z\right\rangle\right|(K)
$$

for any compact set $K \subset \mathbb{R}^{d}$. In addition we have the uniform bound

$$
\sup _{z \in K^{\prime}} \sup _{\delta \in(0, \epsilon)} \int_{K}\left|B_{\delta}^{1}(z)\right|+\left|B_{\delta}^{2}(z)\right| d x \leqslant \sup _{z \in K^{\prime}}|z||E B|(\{x: \operatorname{dist}(x, K) \leqslant \epsilon\})
$$

whenever $K, K^{\prime}$ are compact subsets of $\mathbb{R}^{d}$ and $\epsilon>0$.

Proof. - Let $K$ be a compact subset of $\mathbb{R}^{d}$, and let $B=\left(B^{1}, B^{2}, \ldots, B^{d}\right)$ $\in B D_{\text {loc }}\left(\mathbb{R}^{d}\right)$. Given $z \in \mathbb{R}^{d}$, without loss of generality, we can suppose that $z$ is a unit vector. Up to a rotation we can also assume that $z=e_{d}$, so we can write $x=\left(x^{\prime}, x_{d}\right)$ with $x^{\prime} \in \pi_{d} \sim \mathbb{R}^{d-1}$ (the hyperplane orthogonal to $\left.e_{d}\right)$ and $x_{d} \in \mathbb{R}$. We denote by $K^{d}$ the orthogonal projection of $K$ on $\pi_{d}$ and set $K_{x^{\prime}}^{d}:=\left\{t \in \mathbb{R}:\left(x^{\prime}, t\right) \in K\right\}$. Then we have

$$
\frac{B\left(x^{\prime}, t+\delta\right)-B\left(x^{\prime}, t\right)}{\delta} \cdot e_{d}=\frac{B^{d}\left(x^{\prime}, t+\delta\right)-B^{d}\left(x^{\prime}, t\right)}{\delta} .
$$

By the characterization of $B D$ functions, we know that $B^{d}\left(x^{\prime}, \cdot\right) \in B V_{\text {loc }}(\mathbb{R})$ for $\mathcal{H}^{d-1}$-a.e. $x^{\prime} \in K^{d}$, so, using the result about difference quotients of $B V$ functions of a real variable (see Theorem 2.4 of [3]), we can canonically write

$$
\begin{aligned}
\frac{B^{d}\left(x^{\prime}, t+\delta\right)-B^{d}\left(x^{\prime}, t\right)}{\delta} & =\left(B^{d}\right)_{\delta}^{1}\left(x^{\prime}, t\right)+\left(B^{d}\right)_{\delta}^{2}\left(x^{\prime}, t\right), \\
- & 535-
\end{aligned}
$$


where

$$
\left(B^{d}\right)_{\delta}^{1}\left(x^{\prime}, \cdot\right) \longrightarrow \frac{d}{d t} B^{d}\left(x^{\prime}, \cdot\right) \quad \text { strongly in } L_{\mathrm{loc}}^{1}(\mathbb{R}) \text { as } \delta \downarrow 0
$$

and

$$
\underset{\delta \downarrow 0}{\limsup } \int_{K_{x^{\prime}}^{d}}\left|\left(B^{d}\right)_{\delta}^{2}\left(x^{\prime}, t\right)\right| d t \leqslant\left|D^{s} B^{d}\left(x^{\prime}, \cdot\right)\right|\left(K_{x^{\prime}}^{d}\right) \quad \text { for } \mathcal{H}^{d-1} \text {-a.e. } x^{\prime} \in K^{d} \text {. }
$$

In addition, we have

$$
\sup _{\delta \in(0, \epsilon)} \int_{K_{x^{\prime}}^{d}}\left|\left(B^{d}\right)_{\delta}^{1}\left(x^{\prime}, t\right)\right|+\left|\left(B^{d}\right)_{\delta}^{2}\left(x^{\prime}, t\right)\right| d t \leqslant\left|D B^{d}\left(x^{\prime}, \cdot\right)\right|\left(\left\{t: \operatorname{dist}\left(t, K_{x^{\prime}}^{d}\right)<\epsilon\right\}\right) .
$$

By the structure theorem for $B D$ functions we know that for $\mathcal{H}^{d-1}$-a.e. $x^{\prime} \in K^{d}$ we have

$$
\frac{d}{d t} B^{d}\left(x^{\prime}, t\right)=\frac{\partial B^{d}}{\partial x_{d}}\left(x^{\prime}, t\right)=\left\langle\mathcal{E} B\left(x^{\prime}, t\right) e_{d}, e_{d}\right\rangle \quad \text { for } \mathcal{L}^{1} \text {-a.e. } t \in K_{x^{\prime}}^{d} .
$$

Then, (2.10) and (2.13) yield (2.7), taking also into account (2.12). Analogously, from the identity

$$
\int_{K^{d}}\left|D^{s} B^{d}\left(x^{\prime}, \cdot\right)\right| d \mathcal{H}^{d-1}\left(x^{\prime}\right)=\left|\left\langle E^{s} B e_{d}, e_{d}\right\rangle\right|
$$

and from (2.11) we obtain (2.8), taking again (2.12) into account.

In the following theorem we analyze the behaviour of the commutators

$$
T_{\epsilon}:=(D \cdot(B w)) * \rho_{\epsilon}-D \cdot\left(B\left(w * \rho_{\epsilon}\right)\right),
$$

proving that all limit points as $\epsilon \downarrow 0$ of their modulus are measures singular with respect to $\mathcal{L}^{d}$. In order to give a quantitative estimate we define

$$
I(\rho):=\int_{\mathbb{R}^{d}}|z||\nabla \rho(z)| d z .
$$

TheOrem 2.6 (CONCENTRATion OF COMMUtATORS). Let $B \in B D_{\text {loc }}(\Omega)$ and let $w \in L_{\text {loc }}^{\infty}(\Omega)$. Let $\rho_{\epsilon}$ be a family of mollifiers induced by a radial convolution kernel $\rho$. Then:

(i) The distributions $T_{\epsilon}$ defined by (2.15) are induced by measures with locally uniformly bounded variation in $\Omega$ as $\epsilon \downarrow 0$. 
Traces and fine properties of a $B D$ class of vector fields and applications

(ii) Any limit point, in the distribution sense, of $\left|T_{\epsilon}\right|$ as $\epsilon \downarrow 0$ is a measure $\sigma$ with locally finite variation in $\Omega$ satisfying

$\sigma(A) \leqslant\|w\|_{L^{\infty}(A)}\left(I(\rho)\left|E^{s} B\right|(A)+\left|D^{s} \cdot B\right|(A)\right) \quad$ for any open set $A \subset \subset \Omega$.

Proof. - (i) Let $A \subset \subset \Omega$ and let $\epsilon<\operatorname{dist}(A, \partial \Omega)$. We check first that

$$
T_{\epsilon}=r_{\epsilon} \mathcal{L}^{d}-w * \rho_{\epsilon} D \cdot B \quad \text { in } A,
$$

where

$$
r_{\epsilon}(x):=\int_{\mathbb{R}^{d}} w(y)\left\{(B(x)-B(y)) \cdot \nabla \rho_{\epsilon}(y-x)\right\} d y .
$$

Indeed, for any test function $\varphi \in C_{c}^{\infty}(A)$ we have that $\left\langle T_{\epsilon}, \varphi\right\rangle$ is equal to

$$
\begin{aligned}
& -\int_{\mathbb{R}^{d}} w B \cdot \nabla \rho_{\epsilon} * \varphi d y-\int_{\mathbb{R}^{d}} \varphi B \cdot \nabla \rho_{\epsilon} * w d x-\int_{\mathbb{R}^{d}} w * \rho_{\epsilon} \varphi d D \cdot B \\
= & -\int_{\mathbb{R}^{d}} \int_{\mathbb{R}^{d}} w(y) B(y) \cdot \nabla \rho_{\epsilon}(y-x) \varphi(x) d x d y \\
& -\int_{\mathbb{R}^{d}} \int_{\mathbb{R}^{d}} B(x) \nabla \rho_{\epsilon}(x-y) w(y) \varphi(x) d y d x-\int_{\mathbb{R}^{d}} w * \rho_{\epsilon} \varphi d D \cdot B \\
= & \int_{\mathbb{R}^{d}} r_{\epsilon} \varphi d x-\int_{\mathbb{R}^{d}} w * \rho_{\epsilon} \varphi d D \cdot B
\end{aligned}
$$

(in the last equality we used the fact that $\nabla \rho_{\epsilon}$ is odd). Now, using the fact that $\rho(z)=h(|z|)$ is radial we obtain

$$
r_{\epsilon}(x)=\epsilon^{-1-d} \int_{\mathbb{R}^{d}} w(y) \frac{(B(x)-B(y)) \cdot(y-x)}{|y-x|} h^{\prime}\left(\frac{|y-x|}{\epsilon}\right) d y
$$

and changing variables we get

$$
r_{\epsilon}(x)=\int_{\mathbb{R}^{d}} w(x-\epsilon z) \frac{(B(x-\epsilon z)-B(x)) \cdot z}{\epsilon|z|} h^{\prime}(|z|) d z .
$$

Finally, by integration on $A$, from (2.6) we get the uniform $L^{1}$ bound on $A$ as $\epsilon \downarrow 0$.

(ii) Let $\sigma$ be any limit point of the distributions $\left|T_{\epsilon}\right|$, along some sequence $\epsilon_{i}$, and consider an open set $A \subset \subset \Omega$. By Riesz theorem and (i) we know that $\sigma$ is a measure with locally finite total variation in $\Omega$. Given $\varphi \in C_{c}^{\infty}(A)$ with $0 \leqslant \varphi \leqslant 1$ we have that $\langle\sigma, \varphi\rangle$ is equal to (with the notation $w_{i}=w * \rho_{\epsilon_{2}}$ )

$$
\lim _{i \rightarrow \infty}\left\langle\left|r_{\epsilon_{i}}-w_{i} \operatorname{div} B\right| \mathcal{L}^{d}, \varphi\right\rangle+\left\langle\left|w_{i} D^{s} \cdot B\right|, \varphi\right\rangle
$$


The second term can be estimated from above with $\|w\|_{L^{\infty}(A)}\left|D^{s} \cdot B\right|(A)$ for $i$ large enough. The first term can be estimated using (2.17) and Proposition 2.5 as follows

$$
\begin{aligned}
& \limsup _{i \rightarrow \infty} \int_{A} \varphi(x) \mid \int_{\mathbb{R}^{d}} w\left(x-\epsilon_{i} z\right) \frac{\left(B\left(x-\epsilon_{i} z\right)-B(x)\right) \cdot z}{\epsilon_{i}|z|} h^{\prime}(|z|) \\
& -w_{i}(x) \operatorname{div} B(x) \mid d z d x \\
& \leqslant \limsup _{i \rightarrow \infty} \int_{A} \varphi(x)\left|\int_{\mathbb{R}^{d}} B_{\epsilon_{\imath}}^{1}(-z) w\left(x-\epsilon_{i} z\right) h^{\prime}(|z|)-w(x) \operatorname{div} B(x)\right| d z d x \\
& \quad+\limsup _{i \rightarrow \infty} \varphi(x)\left|\int_{\mathbb{R}^{d}} B_{\epsilon_{\imath}}^{2}(-z) w\left(x-\epsilon_{i} z\right) h^{\prime}(|z|) d z\right| d x .
\end{aligned}
$$

The first limit is equal to 0 because

$$
w\left(x-\epsilon_{i} z\right) B_{\epsilon_{\imath}}^{1}(-z)(x) \longrightarrow w(x)\left\langle\mathcal{E} B(x)(-z), \frac{z}{|z|}\right\rangle \quad \text { strongly in } L_{\mathrm{loc}}^{1}(\Omega)
$$

(taking into account the strong $L_{\text {loc }}^{1}(\Omega)$ convergence of $B_{\epsilon_{i}}^{1}(-z)$ in (2.7) and the strong continuity in $L_{\text {loc }}^{1}$ of translations) so that the limit equals

$$
\int_{A}\left|\varphi(x) w(x)\left(\int_{\mathbb{R}^{d}}\left\langle\mathcal{E} B(x)(-z), \frac{z}{|z|}\right\rangle h^{\prime}(|z|) d z-\operatorname{div} B(x)\right)\right| d x=0,
$$

using the fact that

$$
\begin{aligned}
\int_{\mathbb{R}^{d}}\left\langle\mathcal{E} B(x) z, \frac{z}{|z|}\right\rangle h^{\prime}(|z|) d z & =\int_{\mathbb{R}^{d}}\langle\mathcal{E} B(x) z, \nabla \rho(z)\rangle d z \\
& =-\int_{\mathbb{R}^{d}} \rho(z) \operatorname{div}(\mathcal{E} B(x) z) d z \\
& =-\operatorname{trace} \mathcal{E} B(x)=-\operatorname{div} B(x) .
\end{aligned}
$$

The second limsup can be estimated with $I(\rho)\|w\|_{L^{\infty}(A)}\left|E^{s} B\right|(A)$ using (2.8). Since $\varphi$ is arbitrary we obtain that

$$
\sigma(A) \leqslant\|w\|_{L^{\infty}(A)}\left(I(\rho)\left|E^{s} B\right|(A)+\left|D^{s} \cdot B\right|(A)\right),
$$

and therefore the estimate of the thesis.

\section{Weak traces of vector fields with measure divergence}

In this section we assume that $C: \Omega \subset \mathbb{R}^{d} \rightarrow \mathbb{R}^{d}$ is a locally bounded vector field whose divergence, in the sense of distributions, is a locally finite 
Radon measure in $\Omega$, denoted by $D \cdot C$. We denote by $\mathcal{M}_{\infty}(\Omega)$ the class of these vector fields.

Given a domain with $C^{1}$ boundary $\Omega^{\prime} \subset \subset \Omega$ we can define the trace of the normal component of $C$ on $\partial \Omega^{\prime}$ as a distribution as follows:

$$
\left\langle\operatorname{Tr}\left(C, \partial \Omega^{\prime}\right), \varphi\right\rangle:=\int_{\Omega^{\prime}} \nabla \varphi \cdot C d x+\int_{\Omega^{\prime}} \varphi d D \cdot C \quad \text { for any } \varphi \in C_{c}^{\infty}(\Omega) .
$$

This definition is obviously consistent with the Gauss-Green formula, in the case when $C \in C^{1}\left(\overline{\Omega^{\prime}}, \mathbb{R}^{d}\right)$, and gives that the distribution is induced by the integration on $\partial \Omega^{\prime}$ of $C \cdot \nu_{\Omega^{\prime}}$, where $\nu_{\Omega^{\prime}}$ is the outer normal to $\Omega^{\prime}$. In general it turns out that this distribution is induced by the integration of an $L^{\infty}$ function on $\partial \Omega^{\prime}$, that we will still denote by $\operatorname{Tr}\left(C, \partial \Omega^{\prime}\right)$, and moreover this function depends only on $\partial \Omega^{\prime}$ and its orientation, rather than on $\Omega^{\prime}$. First of all, we need the following approximation lemma.

Lemma 3.1. - Let $\Omega^{\prime} \subset \subset \Omega$ be a domain with $C^{1}$ boundary. For any $\varphi \in C_{c}^{\infty}(\Omega)$ and any $\epsilon>0$ sufficiently small there exists $\tilde{\varphi}_{\epsilon} \in C_{c}^{\infty}(\Omega)$ such that

- $\tilde{\varphi}_{\epsilon}-\varphi$ vanishes in a neighbourhood of $\partial \Omega^{\prime}$,

- $\left\|\tilde{\varphi}_{\epsilon}\right\|_{L^{\infty}\left(\Omega^{\prime}\right)} \leqslant\|\varphi\|_{L^{\infty}\left(\Omega^{\prime}\right)}$,

- $\tilde{\varphi}_{\epsilon}=0$ on $\Omega_{\epsilon}^{\prime}=\left\{x \in \Omega^{\prime}: d\left(x, \partial \Omega^{\prime}\right)>\epsilon\right\}$,

$-\int_{\Omega^{\prime}}\left|\nabla \tilde{\varphi}_{\epsilon}\right| \leqslant \epsilon+\int_{\partial \Omega^{\prime}}|\varphi|$.

Proof. - Since $\partial \Omega^{\prime} \in C^{1}$ it is easy to find (using for instance the fact that $\partial \Omega^{\prime}$ is locally a graph) a family of open sets $\Omega_{h}$ such that $\bar{\Omega}_{h} \subset \Omega^{\prime}$, $\Omega_{h} \uparrow \Omega^{\prime}$ as $h \rightarrow \infty$ and

$$
\limsup _{h \rightarrow \infty}\left|D \chi_{\Omega_{h}}\right|\left(\mathbb{R}^{d}\right) \leqslant\left|D \chi_{\Omega^{\prime}}\right|\left(\mathbb{R}^{d}\right) .
$$

Consider $h$ sufficiently large, so that $\Omega_{h} \supset \overline{\Omega_{\epsilon}^{\prime}}$, and $\eta_{h}=\chi_{\Omega_{h}} * \rho_{\delta}$, with $\delta=\delta(h)>0$ sufficiently small so that $\delta<\operatorname{dist}\left(\partial \Omega_{h}, \partial \Omega^{\prime}\right)$ and the interior of $\left\{\eta_{h}=1\right\}$ contains $\Omega_{\epsilon}^{\prime}$. Setting $\tilde{\varphi}_{h}=\left(1-\eta_{h}\right) \varphi$, it suffices to check that also the last property holds for $h$ large enough. Indeed, by Jensen inequality we still have

$$
\limsup _{h \rightarrow \infty}\left|D \eta_{h}\right|\left(\mathbb{R}^{d}\right) \leqslant\left|D \chi_{\Omega^{\prime}}\right|\left(\mathbb{R}^{d}\right)
$$

and since (by the lower semicontinuity properties of the total variation)

$$
\liminf _{h \rightarrow \infty}\left|D \eta_{h}\right|(A) \geqslant\left|D \chi_{\Omega^{\prime}}\right|(A) \quad \text { for any open set } A \subset \mathbb{R}^{d},
$$


we obtain (see for instance Theorem 1 in $\S 1.9$ of [22]) that $\left|D \eta_{h}\right|$ weakly converge, in the duality with $C_{c}\left(\mathbb{R}^{d}\right)$, to $\left|D \chi_{\Omega^{\prime}}\right|$. We have then

$$
\int_{\Omega^{\prime}}\left|\nabla \tilde{\varphi}_{h}\right| d x \leqslant \int_{\Omega^{\prime}}\left(1-\eta_{h}\right)|\nabla \varphi| d x+\int_{\Omega^{\prime}}|\varphi|\left|\nabla \eta_{h}\right| d x \rightarrow \int_{\partial \Omega^{\prime}}|\varphi| d \mathcal{H}^{d-1} .
$$

Hence we can set $\tilde{\varphi}_{\epsilon}=\tilde{\varphi}_{h}$ for sufficiently large $h$.

Proposition 3.2. - The distribution defined in (3.1) is induced by an $L^{\infty}$ function on $\partial \Omega^{\prime}$, in the following still denoted by $\operatorname{Tr}\left(C, \partial \Omega^{\prime}\right)$, with

$$
\left\|\operatorname{Tr}\left(C, \partial \Omega^{\prime}\right)\right\|_{L^{\infty}\left(\partial \Omega^{\prime}\right)} \leqslant\|C\|_{L^{\infty}\left(\Omega^{\prime}\right)} .
$$

Moreover, if $\Sigma$ is a Borel set contained in $\partial \Omega_{1} \cap \partial \Omega_{2}$ and if $\nu_{\Omega_{1}}=\nu_{\Omega_{2}}$ on $\Sigma$, then

$$
\operatorname{Tr}\left(C, \partial \Omega_{1}\right)=\operatorname{Tr}\left(C, \partial \Omega_{2}\right) \quad \mathcal{H}^{d-1} \text {-a.e. on } \Sigma .
$$

Proof. - First, it is immediate to check that the support of the distribution $\operatorname{Tr}\left(C, \partial \Omega^{\prime}\right)$ is contained in $\partial \Omega^{\prime}$. Let $\epsilon>0$ and $\varphi \in C_{c}^{\infty}(\Omega)$ and take $\tilde{\varphi}_{\epsilon}$ as in the previous lemma. We can estimate in the following way:

$$
\begin{aligned}
\left|\left\langle\operatorname{Tr}\left(C, \partial \Omega^{\prime}\right), \varphi\right\rangle\right| & =\left|\left\langle\operatorname{Tr}\left(C, \partial \Omega^{\prime}\right), \tilde{\varphi}_{\epsilon}\right\rangle\right| \\
& \leqslant\left|\int_{\Omega^{\prime}} \tilde{\varphi}_{\epsilon} d(D \cdot C)\right|+\|C\|_{L^{\infty}\left(\Omega^{\prime}\right)} \int_{\Omega^{\prime}}\left|\nabla \tilde{\varphi}_{\epsilon}\right| \\
& \leqslant\left|\int_{\Omega^{\prime} \backslash \Omega_{\epsilon}^{\prime}} \tilde{\varphi}_{\epsilon} d(D \cdot C)\right|+\|C\|_{L^{\infty}\left(\Omega^{\prime}\right)}\left(\int_{\partial \Omega^{\prime}}|\varphi|+\epsilon\right) \\
& \leqslant\|\varphi\|_{L^{\infty}\left(\Omega^{\prime}\right)}|D \cdot C|\left(\Omega^{\prime} \backslash \Omega_{\epsilon}^{\prime}\right)+\|C\|_{L^{\infty}\left(\Omega^{\prime}\right)}\left(\int_{\partial \Omega^{\prime}}|\varphi|+\epsilon\right) .
\end{aligned}
$$

If $\epsilon \rightarrow 0$ we get $\left|\left\langle\operatorname{Tr}\left(C, \partial \Omega^{\prime}\right), \varphi\right\rangle\right| \leqslant\|C\|_{L^{\infty}\left(\Omega^{\prime}\right)}\|\varphi\|_{L^{1}\left(\partial \Omega^{\prime}\right)}$ and it follows that we can represent $\operatorname{Tr}\left(C, \partial \Omega^{\prime}\right)$ with an $L^{\infty}$ function on $\partial \Omega^{\prime}$.

For the second part of the proposition, let $T_{1}$ and $T_{2}$ be the traces of $C$ on $\partial \Omega_{1}$ and $\partial \Omega_{2}$ respectively. Take $\chi \in C_{c}^{\infty}\left(\mathbb{R}^{d}\right)$ with $0 \leqslant \chi \leqslant 1$ and with support contained in the unit ball $B_{1}$. Take $x \in \Sigma$ satisfying the following two conditions:

(a) $x$ is a Lebesgue point for $T_{1}$ and $T_{2}$,

(b) (2.1) holds at $x$ with $\mu=|D \cdot C|\left\llcorner\left(\Omega_{1} \cup \Omega_{2}\right), E=\Sigma\right.$ and $k=d-1$. 
Observe that these properties are satisfied $\mathcal{H}^{d-1}$-a.e. in $\Sigma$, so it will be enough to show that $T_{1}(x)=T_{2}(x)$ for any such $x$.

Now define $\chi_{\rho}(y)=\chi\left(\frac{y-x}{\rho}\right)$ : we have that the support of $\chi_{\rho}$ is contained in $B_{\rho}(x)$. We are going to use a blow-up argument to show our thesis. If $\rho$ is small enough, we can use $\chi_{\rho}$ as a test function in the definition of trace to obtain

$$
\int_{\partial \Omega_{\imath}} T_{i} \chi_{\rho}=\int_{\Omega_{\imath}} \nabla \chi_{\rho} \cdot C d x+\int_{\Omega_{\imath}} \chi_{\rho} d(D \cdot C) \quad \text { for } i=1,2 .
$$

Let us now estimate the two differences that appear writing explicitly $\int_{\partial \Omega_{1}} T_{1} \chi_{\rho}-\int_{\partial \Omega_{2}} T_{2} \chi_{\rho}$. First of all we have

$$
\left|\int_{\Omega_{1}} \nabla \chi_{\rho} \cdot C-\int_{\Omega_{2}} \nabla \chi_{\rho} \cdot C\right| \leqslant \frac{c}{\rho} \mathcal{L}^{d}\left(\left(\Omega_{1} \triangle \Omega_{2}\right) \cap B_{\rho}(x)\right)=o\left(\rho^{d-1}\right)
$$

because the symmetric difference between $\Omega_{1}$ and $\Omega_{2}$ becomes very small when $\rho \rightarrow 0$. Moreover, we have

$$
\left|\int_{\Omega_{1}} \chi_{\rho} d(D \cdot C)-\int_{\Omega_{2}} \chi_{\rho} d(D \cdot C)\right| \leqslant|D \cdot C|\left(\left(\Omega_{1} \cup \Omega_{2}\right) \cap B_{\rho}(x)\right)=o\left(\rho^{d-1}\right)
$$

because of assumption (b) on $x$.

From these estimates we get

$$
\int_{\partial \Omega_{1}} T_{1} \chi_{\rho}-\int_{\partial \Omega_{2}} T_{2} \chi_{\rho}=o\left(\rho^{d-1}\right) .
$$

Observe that, for $i=1,2$, we have

$\left|\int_{\partial \Omega_{\imath}}\left(T_{i}-T_{i}(x)\right) \chi_{\rho} d \mathcal{H}^{d-1}\right| \leqslant \int_{\partial \Omega_{\imath} \cap B_{\rho}(x)}\left|T_{i}(y)-T_{i}(x)\right| d \mathcal{H}^{d-1}(y)=o\left(\rho^{d-1}\right)$

because $x$ is a Lebesgue point both for $T_{1}$ and $T_{2}$, by assumption (a). Moreover

$$
\frac{1}{\rho^{d-1}} T_{i}(x) \int_{\partial \Omega_{\imath}} \chi_{\rho}(y) d \mathcal{H}^{d-1}(y) \rightarrow T_{i}(x) \int_{\Pi_{x}} \chi(z) d \mathcal{H}^{d-1}(z)
$$

where $\Pi_{x}$ is the tangent plane to $\Sigma$ (that is, to $\partial \Omega_{1}$ and $\partial \Omega_{2}$ ) in $x$. This can be easily seen changing variable, $z=\frac{y-x}{\rho}$, and observing that in a small neighbourhood of $x$ the rescaled sets $\partial \Omega_{\rho}=\left(\partial \Omega_{i}-x\right) / \rho$ both converge in $C^{1}$ to $\Pi_{x}$ as $\rho \downarrow 0$. 
Then, using the triangular inequality, we get

$$
\frac{1}{\rho^{d-1}} \int_{\partial \Omega_{\imath}} T_{i}(y) \chi_{\rho}(y) d \mathcal{H}^{d-1}(y) \rightarrow T_{i}(x) \int_{\Pi_{x}} \chi(z) d \mathcal{H}^{d-1}(z) .
$$

Recalling (3.3) and observing that we can find $\chi$ such that $\int_{\Pi_{x}} \chi(z) d \mathcal{H}^{d-1}(z) \neq 0$ we obtain that $T_{1}(x)=T_{2}(x)$.

We can use the property (3.2) to define the traces $\operatorname{Tr}^{+}(C, \Sigma), \operatorname{Tr}^{-}(C, \Sigma)$ on an oriented $C^{1}$ hypersurface $\Sigma \subset \subset \Omega$. Indeed, choosing an open $C^{1}$ domain $\Omega^{\prime} \subset \subset \Omega$ such that $\Sigma \subset \partial \Omega^{\prime}$ and $\nu_{\Omega^{\prime}}=\nu_{\Sigma}$, we define

$$
\operatorname{Tr}^{-}(C, \Sigma):=\operatorname{Tr}\left(C, \partial \Omega^{\prime}\right) \quad \text { on } \Sigma .
$$

Analogously, choosing this time an open $C^{1}$ domain $\Omega^{\prime \prime} \subset \subset \Omega$ such that $\Sigma \subset \partial \Omega^{\prime \prime}$ and $\nu_{\Omega^{\prime \prime}}=-\nu_{\Sigma}$, we define

$$
\operatorname{Tr}^{+}(C, \Sigma):=-\operatorname{Tr}\left(C, \partial \Omega^{\prime \prime}\right) \quad \text { on } \Sigma .
$$

With the convention that boundaries of open sets are oriented by the outer normal, it turns out that $\operatorname{Tr}^{-}(C, \Sigma)$ is equal on $\Sigma$ to the trace $\operatorname{Tr}\left(C, \partial \Omega^{\prime}\right)$ defined in (3.1). If $\operatorname{Tr}^{+}(C, \Sigma)=\operatorname{Tr}^{-}(C, \Sigma)$ we will sometimes indicate with $\operatorname{Tr}(C, \Sigma)$ the common value.

Definition 3.3 (Normal tRaCE ON COUNTABly $\mathcal{H}^{d-1}$-RECTIFIABle SETS). - Using the previous locality result we can give a meaning to the normal trace of $C$ on any oriented countably $\mathcal{H}^{d-1}$-rectifiable set $\Sigma$. Indeed, we can find countably many oriented $C^{1}$ hypersurfaces $\Sigma_{i}$ and pairwise disjoint Borel sets $E_{i} \subset \Sigma_{i}$ such that $\mathcal{H}^{d}\left(\Sigma \backslash \cup_{i} E_{i}\right)=0$ and $\nu_{\Sigma}(x)$ is the classical normal to $\Sigma_{i}$ for any $x \in E_{i}$, and then we can define

$$
\operatorname{Tr}^{+}(C, \Sigma):=\operatorname{Tr}^{+}\left(C, \Sigma_{i}\right), \quad \operatorname{Tr}^{-}(C, \Sigma):=\operatorname{Tr}^{-}\left(C, \Sigma_{i}\right) \quad \mathcal{H}^{d-1} \text {-a.e. on } E_{i} \text {. }
$$

The locality property ensures that, up to $\mathcal{H}^{d-1}$-negligible sets, this definition does not depend on the choice of $\Sigma_{i}$ and $E_{i}$; nevertheless, as in the case of oriented $C^{1}$ hypersurfaces, it depends on the orientation.

Proposition 3.4. - Let $C$ be a vector field in $\mathcal{M}_{\infty}(\Omega)$. Then:

(i) $|D \cdot C|(B)=0$ for any $\mathcal{H}^{d-1}$-negligible set $B \subset \Omega$;

(ii) for any $C^{1}$ oriented hypersurface $\Sigma \subset \Omega$ we have

$$
D \cdot C\left\llcorner\Sigma=\left(\operatorname{Tr}^{+}(C, \Sigma)-\operatorname{Tr}^{-}(C, \Sigma)\right) \mathcal{H}^{d-1}\llcorner\Sigma .\right.
$$


Proof. - (i) By inner regularity it is enough to show the thesis for any compact set $K \subset \Omega$ such that $\mathcal{H}^{d-1}(K)=0$. Then we will show that

$$
\left\langle\chi_{K} D \cdot C, \varphi\right\rangle=0 \quad \text { for any } \varphi \in C_{c}^{\infty}(\Omega) .
$$

Fix any $\epsilon>0$ and take a finite family of balls $\left\{B_{i}\right\}$ such that $K \subset \cup_{i} B_{i}$ and $\sum_{i} \mathcal{H}^{d-1}\left(\partial B_{i}\right)<\epsilon$. Let $\chi_{\epsilon}$ be the characteristic function of $\cup_{i} B_{i}$ and let $\chi_{\epsilon, \delta}=\chi_{\epsilon} * \rho_{\delta}$ with $\rho_{\delta}$ a standard convolution kernel. If $\delta$ is small enough, we can suppose that the supports of all $\chi_{\epsilon, \delta}$ are contained in a compact set $\tilde{K} \subset \Omega$. Then we have

$$
\left\langle\chi_{\epsilon, \delta} D \cdot C, \varphi\right\rangle=-\int_{\Omega} C \cdot \nabla\left(\chi_{\epsilon, \delta} \varphi\right) d x=-\int_{\Omega} \varphi C \cdot \nabla \chi_{\epsilon, \delta} d x-\int_{\Omega} \chi_{\epsilon, \delta} C \cdot \nabla \varphi d x
$$

and a simple estimate gives

$$
\begin{gathered}
\left|\left\langle\chi_{\epsilon, \delta} D \cdot C, \varphi\right\rangle\right| \\
\leqslant\|C\|_{L^{\infty}(\tilde{K})}\left[\|\varphi\|_{L^{\infty}(\tilde{K})} \int_{\Omega}\left|\nabla \chi_{\epsilon, \delta}\right| d x+\|\nabla \varphi\|_{L^{\infty}(\tilde{K})} \mathcal{L}^{d}\left(\left\{\chi_{\epsilon, \delta}>0\right\}\right)\right] .
\end{gathered}
$$

Since $\nabla \chi_{\epsilon, \delta}=D \chi_{\epsilon} * \rho_{\delta}$, Jensen's inequality gives

$$
\limsup _{\epsilon \downarrow 0} \limsup _{\delta \downarrow 0} \int_{\Omega}\left|\nabla \chi_{\epsilon, \delta}\right| d x \leqslant \limsup _{\epsilon \downarrow 0}\left|D \chi_{\epsilon}\right|(\Omega)=0 .
$$

Hence we can apply the estimate above with a suitable $\delta(\epsilon) \rightarrow 0$ to obtain (3.4).

(ii) Since the statement has a local nature we can test the identity with $\varphi \in C_{c}^{\infty}(A)$, where $A \subset \subset \Omega$ and $\Sigma$ is equal in $A$ to the 0 level set of $F \in C^{1}(\bar{A})$ with $|\nabla F|>0$ in $A$. Setting $A^{+}=A \cap\{F>0\}$ and $A^{-}=$ $A \cap\{F<0\}$ and using the definition of $\operatorname{Tr}^{+}(C, \Sigma)$ and $\operatorname{Tr}^{-}(C, \Sigma)$, we get

$$
-\int_{\Sigma} \operatorname{Tr}^{+}(C, \Sigma) \varphi d \mathcal{H}^{d-1}=\int_{A^{+}} \nabla \varphi \cdot C d x+\int_{A^{+}} \varphi d(D \cdot C)
$$

and

$$
\int_{\Sigma} \operatorname{Tr}^{-}(C, \Sigma) \varphi d \mathcal{H}^{d-1}=\int_{A^{-}} \nabla \varphi \cdot C d x+\int_{A^{-}} \varphi d(D \cdot C) .
$$

Taking into account that $\int_{A} \nabla \varphi \cdot C d x=-\int_{A} \varphi d D \cdot C$, because $\varphi \in C_{c}^{\infty}(A)$, adding up these two equalities we get

$$
\int_{\Sigma} \varphi d(D \cdot C)=\int_{\Sigma}\left[\operatorname{Tr}^{+}(C, \Sigma)-\operatorname{Tr}^{-}(C, \Sigma)\right] \varphi d \mathcal{H}^{d-1} .
$$


DEFINITION 3.5 (FAMILIES OF LEVEL SURFACES AND GRAPHS). - Let $I \subset \mathbb{R}$ be an open interval and let $\Sigma_{t}, t \in I$, be a family of oriented hypersurfaces. We say that $\left\{\Sigma_{t}\right\}_{t \in I}$ is a family of level surfaces in $\Omega^{\prime} \subset \subset \Omega$ if there exists $F \in C^{1}\left(\overline{\Omega^{\prime}}\right)$ such that $F\left(\Omega^{\prime}\right)=I,\{F=t\}=\Sigma_{t}$ for any $t \in I$, $|\nabla F|>0$ in $\Omega^{\prime}$ and $\Sigma_{t}$ is oriented by $\nabla F /|\nabla F|$.

We say that $\left\{\Sigma_{t}\right\}_{t \in I}$ is a family of graphs in $\Omega^{\prime} \subset \subset \Omega$ if, in addition, in a suitable system of coordinates, we have

$$
\Omega^{\prime}=\left\{x \in \mathbb{R}^{d}:\left(x_{1}, \ldots, x_{d-1}\right) \in D, f\left(x_{1}, \ldots, x_{d-1}\right)-x_{d} \in I\right\}
$$

for some open set $D \subset \mathbb{R}^{d-1}$ and some $f \in C^{1}(D)$, and

$$
F\left(x_{1}, \ldots, x_{d}\right)=f\left(x_{1}, \ldots, x_{d-1}\right)-x_{d} \quad \forall x \in \Omega^{\prime} .
$$

The following proposition shows that the weak trace is generically consistent with the pointwise values of the vector field on families of level surfaces.

Proposition 3.6. - Let $C \in \mathcal{M}_{\infty}(\Omega)$ and let $\Sigma_{t}, t \in I$, be a family of level surfaces contained in $\Omega^{\prime} \subset \subset \Omega$ as in Definition 3.5. Then

$$
C \cdot \nu_{\Sigma_{t}}=\operatorname{Tr}^{+}\left(C, \Sigma_{t}\right)=\operatorname{Tr}^{-}\left(C, \Sigma_{t}\right) \quad \mathcal{H}^{d-1} \text {-a.e. on } \Sigma_{t} \text {, for } \mathcal{L}^{1} \text {-a.e. } t \in I \text {. }
$$

Proof. - Since $D \cdot C$ is locally finite, there exists $N_{1} \subset I$ at most countable such that $D \cdot C\left\llcorner\Sigma_{t}=0\right.$ for all $t \in I \backslash N_{1}$. For these values of $t$, using Proposition 3.4, we have $\operatorname{Tr}^{+}\left(C, \Sigma_{t}\right)=\operatorname{Tr}^{-}\left(C, \Sigma_{t}\right)$.

Let us now approximate $C$ with smooth vector fields $C_{\epsilon}=C * \rho_{\epsilon}$ by convolution, so that $C_{\epsilon}$ are locally uniformly bounded in $L^{\infty}$ and converge to $C$ in $L_{\text {loc }}^{1}(\Omega)$. Since $D \cdot C_{\epsilon}=(D \cdot C) * \rho_{\epsilon}$, by a general property of convolutions of measures we have that $\left|D \cdot C_{\epsilon}\right|$ weakly*-converge, in the duality with $C_{c}(\Omega)$, to $|D \cdot C|$. Hence (see for instance Proposition 1.62(b) of [8]) we have also $D \cdot C_{\epsilon} \rightarrow D \cdot C$ weakly*, in the duality with continuous and bounded functions in $\tilde{\Omega}$, for every open set $\tilde{\Omega} \subset \subset \Omega$ such that $|D \cdot C|(\partial \tilde{\Omega})=0$. For such an $\tilde{\Omega}$, using the definition of trace we have

$$
\operatorname{Tr}\left(C_{\epsilon}, \partial \tilde{\Omega}\right) \rightarrow \operatorname{Tr}(C, \partial \tilde{\Omega})
$$

in the sense of distributions on $\Omega$. Observing that $\operatorname{Tr}\left(C_{\epsilon}, \partial \tilde{\Omega}\right)$ are uniformly bounded in $L^{\infty}(\partial \tilde{\Omega})$ as $\epsilon \downarrow 0$, we deduce that we have also convergence weak $^{*}$ in $L^{\infty}(\partial \tilde{\Omega})$. Noticing that, for every $t \in I \backslash N_{1}$, we can find an open domain $\tilde{\Omega}$ with $\Sigma_{t} \subset \partial \tilde{\Omega}$ and $|D \cdot C|(\partial \tilde{\Omega})=0$, we easily deduce that

$$
\operatorname{Tr}\left(C_{\epsilon}, \Sigma_{t}\right) \rightarrow \operatorname{Tr}\left(C, \Sigma_{t}\right) \quad \text { weakly* in } L^{\infty}\left(\Sigma_{t}\right) \text { for any } t \in I \backslash N_{1} .
$$


Traces and fine properties of a $B D$ class of vector fields and applications

Recalling that $\Omega^{\prime} \subset \subset \Omega$, we can extract a "fastly converging" subsequence $C_{\epsilon_{h}}$, i.e. a subsequence such that $\sum_{h}\left\|C_{\epsilon_{h}}-C\right\|_{L^{1}\left(\Omega^{\prime}\right)}<+\infty$. Then we have

$$
\begin{aligned}
\int_{I} \sum_{h}\left\|C_{\epsilon_{h}}-C\right\|_{L^{1}\left(\Sigma_{t}\right)} d t & =\sum_{h} \int_{I}\left\|C_{\epsilon_{h}}-C\right\|_{L^{1}\left(\Sigma_{t}\right)} d t \\
& =\sum_{h} \int_{\Omega^{\prime}}\left|\nabla F \| C_{\epsilon_{h}}-C\right| d x<+\infty
\end{aligned}
$$

and it follows that $\sum_{h}\left\|C_{\epsilon_{h}}-C\right\|_{L^{1}\left(\Sigma_{t}\right)}<+\infty$ for all $t \in I \backslash N_{2}$, with $\mathcal{L}^{1}\left(N_{2}\right)=0$. Clearly this means that

$$
\left.\left.C_{\epsilon_{h}}\right|_{\Sigma_{t}} \rightarrow C\right|_{\Sigma_{t}} \quad \text { in } L^{1}\left(\Sigma_{t}, \mathcal{H}^{d-1}\left\llcorner\Sigma_{t}\right)\right.
$$

for all $t \in I \backslash N_{2}$. Recalling that for smooth vector fields the trace is the classical one, we get the desired result for all $t \in I \backslash\left(N_{1} \cup N_{2}\right)$.

Finally, in the more particular framework of families of graphs (with the same notation introduced in Definition 3.5), we investigate the continuity of the maps $t \mapsto \operatorname{Tr}^{+}\left(C, \Sigma_{t}\right)$ and $t \mapsto \operatorname{Tr}^{-}\left(C, \Sigma_{t}\right)$. Looking at the traces as functions on $D$, it turns out that the maps are weakly* continuous but not strongly continuous in general.

Theorem 3.7 (WeAK* CONTINUITY OF TRACES). - Let $\Sigma_{t}, t \in I$, be a family of graphs as in Definition 3.5 and let $C \in \mathcal{M}_{\infty}(\Omega)$. Fix $t_{0} \in I$ and set

$$
\begin{gathered}
\bar{\alpha}\left(x^{\prime}\right)=\operatorname{Tr}^{-}\left(C, \Sigma_{t_{0}}\right)\left(x^{\prime}, f\left(x^{\prime}\right)-t_{0}\right), \\
\alpha_{t}\left(x^{\prime}\right)=\operatorname{Tr}^{+}\left(C, \Sigma_{t}\right)\left(x^{\prime}, f\left(x^{\prime}\right)-t\right) \quad \text { for } t>t_{0} .
\end{gathered}
$$

Then we have

$$
\lim _{t \downarrow t_{0}} \alpha_{t}\left(x^{\prime}\right)=\bar{\alpha}\left(x^{\prime}\right) \quad \text { weakly in } L^{\infty}\left(D, \mathcal{L}^{d-1}\llcorner D) .\right.
$$

Proof. - Let $\varphi \in C_{c}^{\infty}(D)$ and set $\Phi\left(x^{\prime}, x_{d}\right)=\varphi\left(x^{\prime}\right)$ and

$$
\Omega_{t}=\left\{\left(x^{\prime}, x_{d}\right): x^{\prime} \in D, f\left(x^{\prime}\right)-t<x_{d}<f\left(x^{\prime}\right)-t_{0}\right\} .
$$

Observing that we can test against $\Phi$ in the trace formula and estimating in the obvious way, we get

$$
\begin{aligned}
&\left|\int_{\partial \Omega_{t}}\left[\operatorname{Tr}^{+}\left(C, \Sigma_{t}\right)(x) \Phi(x)-\operatorname{Tr}^{-}\left(C, \Sigma_{t_{0}}\right)(x) \Phi(x)\right] d \mathcal{H}^{d-1}(x)\right| \\
&=\left|-\int_{\Omega_{t}} \nabla \Phi \cdot C d x-\int_{\Omega_{t}} \Phi d(D \cdot C)\right| \\
& \leqslant\|\nabla \Phi\|_{L^{\infty}\left(\Omega_{t}\right)}\|C\|_{L^{\infty}\left(\Omega_{t}\right)} \mathcal{L}^{d}\left(\Omega_{t}\right)+\|\Phi\|_{L^{\infty}\left(\Omega_{t}\right)}|D \cdot C|\left(\Omega_{t}\right), \\
&-545-
\end{aligned}
$$


that clearly vanishes as $t \downarrow t_{0}$. This shows that

$$
\int_{D} \alpha_{t}\left(x^{\prime}\right) \sigma\left(x^{\prime}\right) \varphi\left(x^{\prime}\right) d x^{\prime} \rightarrow \int_{D} \bar{\alpha}\left(x^{\prime}\right) \sigma(x) \varphi\left(x^{\prime}\right) d x^{\prime}
$$

(where $\sigma\left(x^{\prime}\right)=\sqrt{1+\left|\nabla f\left(x^{\prime}\right)\right|^{2}}$ is the area element) for any $\varphi \in C_{c}^{\infty}(D)$. Thanks to the density of smooth functions and to the uniform boundedness of $\alpha_{t}$ it follows that

$$
\lim _{t \downarrow t_{0}} \alpha_{t}\left(x^{\prime}\right) \sigma\left(x^{\prime}\right)=\bar{\alpha}\left(x^{\prime}\right) \sigma\left(x^{\prime}\right) \quad \text { weakly* in } L^{\infty}\left(D, \mathcal{L}^{d-1}\llcorner D)\right.
$$

and observing that $\sigma\left(x^{\prime}\right) \geqslant 1$ we get the desired convergence.

Obviously a similar result holds in the case of convergence of $\operatorname{Tr}^{-}\left(C, \Sigma_{t}\right)$ to $\operatorname{Tr}^{+}\left(C, \Sigma_{t_{0}}\right)$ as $t \uparrow t_{0}$. Let us now show that $w^{*}$-continuity is the best we can hope for. The following example is taken from [11].

Example 3.8. - Set $\Omega=\mathbb{R}^{2}$ and $\Omega^{\prime}$ the unit ball. Consider the horizontal stripes $\Omega_{j}^{+}=\left\{\frac{1}{2^{\jmath+1}}<y<\frac{1}{2^{\jmath}}\right\}$ and $\Omega_{j}^{-}=\left\{-\frac{1}{2^{\jmath}}<y<-\frac{1}{2^{j+1}}\right\}$ for $j$ positive integer. Then divide each stripe into squares and consider in each square the vector field (of unitary modulus and constant in each triangle) drawn in the figure. Observing that the discontinuities of the vector field are always parallel to the discontinuities lines, thanks to Proposition 3.4 we deduce that it is divergence-free, so it clearly belongs to $\mathcal{M}_{\infty}(\Omega)$. It is also immediate to check that the trace on $\{y=0\}$ vanishes, but in this case we cannot expect strong $L^{1}$ convergence to 0 of the traces on the horizontal lines, due to the oscillations of the field.

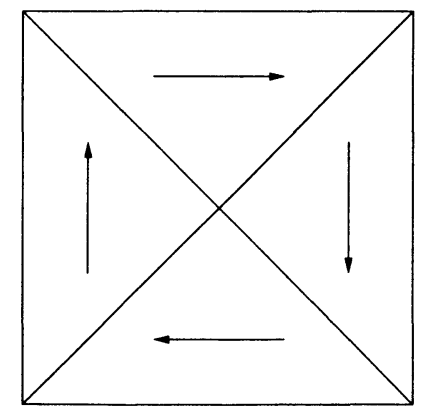

\section{Chain rule for traces on hypersurfaces}

In this section we assume that $C \in \mathcal{M}_{\infty}(\Omega)$ has the form $w B$ with $w: \Omega \rightarrow \mathbb{R}, B: \Omega \rightarrow \mathbb{R}^{d}$, both locally bounded, with $B \in B D_{\text {loc }}(\Omega)$. We 
show first that this class of vector fields is stable under "renormalization" of the scalar component $w$.

LEMMA 4.1 (WEAK RENORMALIZATION). - Under the assumptions above, we have $h(w) B \in \mathcal{M}_{\infty}(\Omega)$ for any $h \in C^{1}(\mathbb{R})$. Furthermore, for any open set $\Omega^{\prime} \subset \subset \Omega$ we have

$$
\begin{aligned}
|D \cdot(h(w) B)| \leqslant & \sup _{t \in[-M, M]}\left|h^{\prime}(t)\right|\left(M I(\rho)\left|E^{s} B\right|+M\left|D^{s} \cdot B\right|+|D \cdot(w B)|\right) \\
& +\sup _{t \in[-M, M]}\left|t h^{\prime}(t)-h(t)\right||D \cdot B| \quad \text { in } \Omega^{\prime},
\end{aligned}
$$

with $M=\|w\|_{L^{\infty}\left(\Omega^{\prime}\right)}$ and $I(\rho)$ defined as in (2.16).

Proof. - Let $T_{\epsilon}$ be defined as in (2.15) with $w_{\epsilon}=w * \rho_{\epsilon}$, so that

$$
T_{\epsilon}=(D \cdot(B w)) * \rho_{\epsilon}-B \cdot \nabla w_{\epsilon} \mathcal{L}^{d}-w_{\epsilon} D \cdot B .
$$

Multiplying both sides by $h^{\prime}\left(w_{\epsilon}\right)$ we obtain

$D \cdot\left(h\left(w_{\epsilon}\right) B\right)=h^{\prime}\left(w_{\epsilon}\right)(D \cdot(B w)) * \rho_{\epsilon}+\left(h\left(w_{\epsilon}\right)-h^{\prime}\left(w_{\epsilon}\right) w_{\epsilon}\right) D \cdot B-h^{\prime}\left(w_{\epsilon}\right) T_{\epsilon}$.

Passing to the limit as $\epsilon \downarrow 0$, the thesis is achieved using Theorem 2.6(ii).

The following theorem is one of the main results of the paper: due to the nonlinearities involved its proof cannot be achieved using only the $w^{*}$-continuity properties of the trace operator. The proof involves a suitable extension argument, based on Gagliardo's theorem, and the quantitative estimate in Lemma 4.1.

Theorem 4.2 (Change of VARIABles for TraCes). - Let $\Omega^{\prime} \subset \subset \Omega$ be an open domain with a $C^{1}$ boundary and let $h \in C^{1}(\mathbb{R})$. Then

$$
\operatorname{Tr}\left(h(w) B, \partial \Omega^{\prime}\right)=h\left(\frac{\operatorname{Tr}\left(w B, \partial \Omega^{\prime}\right)}{\operatorname{Tr}\left(B, \partial \Omega^{\prime}\right)}\right) \operatorname{Tr}\left(B, \partial \Omega^{\prime}\right) \quad \mathcal{H}^{d-1} \text {-a.e. on } \partial \Omega^{\prime},
$$

where the ratio $\operatorname{Tr}\left(w B, \partial \Omega^{\prime}\right) / \operatorname{Tr}\left(B, \partial \Omega^{\prime}\right)$ is arbitrarily defined at points where the trace $\operatorname{Tr}\left(B, \partial \Omega^{\prime}\right)$ vanishes.

Proof. - It is not restrictive to assume that the larger open set $\Omega$ is bounded and that it has a $C^{1}$ boundary.

Step 1. Let $\Omega^{\prime \prime}=\Omega \backslash \overline{\Omega^{\prime}}$. In this step we prove that

$$
\operatorname{Tr}\left(h(w) B, \partial \Omega^{\prime \prime}\right)=h\left(\frac{\operatorname{Tr}\left(w B, \partial \Omega^{\prime \prime}\right)}{\operatorname{Tr}\left(B, \partial \Omega^{\prime \prime}\right)}\right) \operatorname{Tr}\left(B, \partial \Omega^{\prime \prime}\right) \quad \mathcal{H}^{d-1} \text {-a.e. on } \partial \Omega^{\prime \prime},
$$


under the assumption that both $w$ and the components of $B$ are bounded and belong to the Sobolev space $W^{1,1}\left(\Omega^{\prime \prime}\right)$. Indeed, the identity is trivial if both $w$ and $B$ are continuous up to the boundary, and the proof of the general case can be immediately achieved by a density argument based on the strong continuity of the trace operator from $W^{1,1}\left(\Omega^{\prime \prime}\right)$ to $L^{1}\left(\partial \Omega^{\prime \prime}, \mathcal{H}^{d-1}\left\llcorner\partial \Omega^{\prime \prime}\right)\right.$ (see for instance Theorem 3.88 of $[8]$ ).

Step 2. In this step we prove the general case. Let us apply Gagliardo's theorem on the surjectivity of the trace operator from $W^{1,1}$ into $L^{1}$ to obtain a bounded vector field $B_{1} \in\left[W^{1,1}\left(\Omega^{\prime \prime}\right)\right]^{d}$ whose trace on $\partial \Omega^{\prime} \subset \partial \Omega^{\prime \prime}$ is equal to the trace of $B$, seen as a function in $B D\left(\Omega^{\prime}\right)$. In particular $\operatorname{Tr}\left(B, \partial \Omega^{\prime}\right)=-\operatorname{Tr}\left(B_{1}, \partial \Omega^{\prime \prime}\right)$ on $\partial \Omega^{\prime}$. Defining

$$
\tilde{B}(x):= \begin{cases}B(x) & \text { if } x \in \Omega^{\prime} \\ B_{1}(x) & \text { if } x \in \Omega^{\prime \prime}\end{cases}
$$

it turns out that $\tilde{B} \in B D_{\mathrm{loc}}(\Omega)$ and that (see (2.5))

$$
|E \tilde{B}|\left(\partial \Omega^{\prime}\right)=0 .
$$

Let us consider the function $\theta:=\operatorname{Tr}\left(w B, \partial \Omega^{\prime}\right) / \operatorname{Tr}\left(B, \partial \Omega^{\prime}\right)$ (set equal to 0 wherever the denominator is 0$)$ and let us prove that $\|\theta\|_{L^{\infty}\left(\partial \Omega^{\prime}\right)}$ is less than $\|w\|_{L^{\infty}\left(\Omega^{\prime}\right)}$. Indeed, writing $\partial \Omega^{\prime}$ as the 0-level set of a $C^{1}$ function $F$ with $|\nabla F|>0$ on $\partial \Omega^{\prime}$ and $\{F=t\} \subset \Omega^{\prime}$ for $\mathcal{L}^{1}$-a.e. $t>0$ sufficiently small, by Proposition 3.6 we have

$$
\begin{aligned}
-\|w\|_{L^{\infty}\left(\Omega^{\prime}\right)} \operatorname{Tr}(B, \partial\{F>t\}) & \leqslant \operatorname{Tr}(w B, \partial\{F>t\}) \\
& \leqslant\|w\|_{L^{\infty}\left(\Omega^{\prime}\right)} \operatorname{Tr}(B, \partial\{F>t\})
\end{aligned}
$$

$\mathcal{H}^{d-1}$-a.e. on $\{F=t\}$. Passing to the limit as $t \downarrow 0$ and using Theorem 3.7 we recover the same inequality on $\{F=0\}$, proving the boundedness of $\theta$.

Now, still using Gagliardo's theorem, we can find a bounded function $w_{1} \in W^{1,1}\left(\Omega^{\prime \prime}\right)$ whose trace on $\partial \Omega^{\prime}$ is given by $\theta$, so that the normal trace of $w_{1} B_{1}$ on $\partial \Omega^{\prime \prime}$ is equal to $-\operatorname{Tr}\left(w B, \partial \Omega^{\prime}\right)$ on the whole of $\partial \Omega^{\prime}$. Defining

$$
\tilde{w}(x):= \begin{cases}w(x) & \text { if } x \in \Omega^{\prime} \\ w_{1}(x) & \text { if } x \in \Omega^{\prime \prime}\end{cases}
$$

by Proposition 3.4 we obtain

$$
|D \cdot(\tilde{w} \tilde{B})|\left(\partial \Omega^{\prime}\right)=0 .
$$

Let us apply now Lemma 4.1 and (4.2), (4.3), to obtain that the divergence of the vector field $h(\tilde{w}) \tilde{B}$ is a measure with finite total variation in $\Omega$, 
whose restriction to $\partial \Omega^{\prime}$ vanishes. As a consequence, Proposition 3.4 gives

$$
\operatorname{Tr}^{+}\left(h(\tilde{w}) \tilde{B}, \partial \Omega^{\prime}\right)=\operatorname{Tr}^{-}\left(h(\tilde{w}) \tilde{B}, \partial \Omega^{\prime}\right) \quad \mathcal{H}^{d-1} \text {-a.e. on } \partial \Omega^{\prime} .
$$

By applying (4.4), Step 1, and finally our choice of $B_{1}$ and $w_{1}$ the following chain of equalities holds $\mathcal{H}^{d-1}$-a.e. on $\partial \Omega^{\prime}$ :

$$
\begin{aligned}
\operatorname{Tr}\left(h(w) B, \partial \Omega^{\prime}\right) & =\operatorname{Tr}^{-}\left(h(\tilde{w}) \tilde{B}, \partial \Omega^{\prime}\right) \\
& =\operatorname{Tr}^{+}\left(h(\tilde{w}) \tilde{B}, \partial \Omega^{\prime}\right) \\
& =-\operatorname{Tr}\left(h\left(w_{1}\right) B_{1}, \partial \Omega^{\prime \prime}\right) \\
& =-h\left(\frac{\operatorname{Tr}\left(w_{1} B_{1}, \partial \Omega^{\prime \prime}\right)}{\operatorname{Tr}\left(B_{1}, \partial \Omega^{\prime \prime}\right)}\right) \operatorname{Tr}\left(B_{1}, \partial \Omega^{\prime \prime}\right) \\
& =h\left(\frac{\operatorname{Tr}\left(w B, \partial \Omega^{\prime}\right)}{\operatorname{Tr}\left(B, \partial \Omega^{\prime}\right)}\right) \operatorname{Tr}\left(B, \partial \Omega^{\prime}\right)
\end{aligned}
$$

\section{The Cauchy problem with a $S B D$ velocity field}

In this section we show that any distributional solution of the transport equation $B \cdot \nabla w=c \mathcal{L}^{d}$ is renormalizable, according to the terminology introduced by Di Perna and Lions in [21], when the vector field $B$ belongs to $S B D_{\text {loc }}(\Omega)$ and $D \cdot B \ll \mathcal{L}^{d}$. In the rest of the section we illustrate several by now standard consequences of this fact, based on the tools introduced in [21] and in [3].

Theorem 5.1 (Renormalization Lemma). - Let $B \in S B D_{\text {loc }}(\Omega)$ be a locally bounded vector field with $D \cdot B \ll \mathcal{L}^{d}$. If $w$ is a locally bounded function satisfying $D \cdot(w B) \ll \mathcal{L}^{d}$, then

$$
D \cdot(h(w) B)=-L h(w) D \cdot B+h^{\prime}(w) D \cdot(w B),
$$

with $\operatorname{Lh}(t)=t h^{\prime}(t)-h(t)$ for any $h \in C^{1}(\mathbb{R})$.

Proof. - Passing to the limit as $\epsilon \downarrow 0$ in (4.1) of Lemma 4.1 and using Theorem 2.6(ii) we obtain

$$
D \cdot(h(w) B)=-L h(w) D \cdot B+h^{\prime}(w) D \cdot(w B)+\sigma
$$

for some measure $\sigma$ absolutely continuous with respect to $\left|E^{s} B\right|$. By the $S B D$ assumption the measure $\left|E^{s} B\right|$ is concentrated on some countably $\mathcal{H}^{d-1}$-rectifiable set $\Sigma$. Therefore, it suffices to show that

$$
\begin{gathered}
D \cdot(h(w) B)\llcorner\Sigma=0 . \\
-549-
\end{gathered}
$$


By the rectifiability of $\Sigma$ we can cover $\mathcal{H}^{d-1}$-almost all of $\Sigma$ by compact sets $K_{i}$, each contained in a $C^{1}$ oriented hypersurface $\partial \Omega_{i}$. Taking into account the first statement in Proposition 3.4, it suffices to show that $D \cdot(h(w) B)\left\llcorner\partial \Omega_{i}=0\right.$ for any $i$. Since the divergences of $B$ and $w B$ are absolutely continuous the second statement in Proposition 3.4 gives

$$
\operatorname{Tr}^{+}\left(B, \partial \Omega_{i}\right)=\operatorname{Tr}^{-}\left(B, \partial \Omega_{i}\right) \text { and } \operatorname{Tr}^{+}\left(w B, \partial \Omega_{i}\right)=\operatorname{Tr}^{-}\left(w B, \partial \Omega_{i}\right)
$$

$\mathcal{H}^{d-1}$-a.e. in $\partial \Omega_{i}$, so that the change of variables formula for traces gives

$$
\operatorname{Tr}^{+}\left(h(w) B, \partial \Omega_{i}\right)=\operatorname{Tr}^{-}\left(h(w) B, \partial \Omega_{i}\right) .
$$

From Proposition 3.4 again we obtain that $D \cdot(h(w) B)\left\llcorner\partial \Omega_{i}=0\right.$.

Introducing the notation (justified by the absolute continuity assumption on the divergence)

$$
\langle B \cdot \nabla w, \varphi\rangle:=\langle D \cdot(w B), \varphi\rangle-\langle w D \cdot B, \varphi\rangle \quad \varphi \in C_{c}^{\infty}\left(\mathbb{R}^{d}\right)
$$

we can read the renormalization lemma in an easier way:

$$
B \cdot \nabla w=c \mathcal{L}^{d} \quad \Longrightarrow \quad B \cdot \nabla(h(w))=c h^{\prime}(w) \mathcal{L}^{d} \quad \text { for any } h \in C^{1}(\mathbb{R}) .
$$

Notice that in the general case $B \in B D_{\text {loc }}(\Omega)$ and $D \cdot B \ll \mathcal{L}^{d}$ the previous argument still gives that the absolutely continuous part of $B$. $\nabla(h(w))$ is $h^{\prime}(w) B \cdot \nabla w$ and that $B \cdot \nabla(h(w))\llcorner\Sigma=0$ for any countably $\mathcal{H}^{d-1}$-rectifiable set, but these informations are not sufficient to conclude, as $B \cdot \nabla(h(w))$ could a priori have also a "Cantor" part, neither absolutely continuous with respect to $\mathcal{L}^{d}$, nor concentrated on rectifiable sets.

In the final part of this section we briefly recall the consequences of the renormalization property for $S B D$ functions, referring to [3] for more details, since the proofs here will be omitted because they are just the same ones we have in the $B V$ case.

First we can apply the renormalization property to derive uniqueness and comparison results for bounded weak solutions of the transport equation $\partial_{t} w+b \cdot \nabla w=c \mathcal{L}^{d+1}$ in the autonomous case and also in the nonautonomous case, when $b$ is $S B D$ with respect to the spatial variables.

We fix $T \in(0,+\infty)$, and consider a vector field $B$ of the form $B=(1, b)$, with a function $b(t, x):(0, T) \times \mathbb{R}^{d} \rightarrow \mathbb{R}^{d}$ satisfying the following conditions

$$
b_{t}(x)=b(t, x) \in S B D_{\text {loc }}\left(\mathbb{R}^{d}\right) \text { for } \mathcal{L}^{1} \text {-a.e. } t \in(0, T),
$$


Traces and fine properties of a $B D$ class of vector fields and applications

$\int_{(0, T) \times B_{R}}|b| d t d x+\int_{I}\left|E b_{t}\right|\left(B_{R}\right) d t<+\infty \quad$ whenever $R>0$ and $\bar{I} \subset(0, T)$.

We also assume that for $\mathcal{L}^{1}$-a.e. $t \in(0, T)$ the distribution $D \cdot b_{t}$ is representable as $\operatorname{div} b_{t} \mathcal{L}^{d}$ with

$$
\int_{0}^{T}\left\|\operatorname{div} b_{t}\right\|_{L^{\infty}\left(B_{R}\right)} d t<+\infty \quad \text { whenever } R>0 .
$$

With the particular choice of $B=(1, b)$ the renormalization property, which can be proved along the same lines of Theorem 5.1 by a mollification along the spatial variables only, reads as follows:

$B \cdot \nabla_{t, x} w=c \mathcal{L}^{d+1} \Longrightarrow B \cdot \nabla_{t, x}(h(w))=c h^{\prime}(w) \mathcal{L}^{d+1} \quad$ for any $h \in C^{1}(\mathbb{R})$.

Moreover the equation on the left hand side reduces to a transport equation of the form

$$
\frac{\partial w_{t}}{\partial t}+b_{t} \cdot \nabla_{x} w_{t}=c_{t} \quad \text { in }(0, T) \times \mathbb{R}^{d}
$$

(with $w_{t}=w(t, \cdot), c_{t}=c(t, \cdot)$ ). When $c_{t}=e_{t} w_{t}$ for this PDE the same comparison result that the first author proved for $B V$ functions in [3] holds (the proof uses Gronwall inequality and (5.4)). For the reader's convenience we recall the statement of the theorem.

THEOREM 5.2 (COMPARISON PROPERTY). - Let $w_{t}^{i}, i=1,2$, be solutions of the transport equation (5.5) in $(0, T) \times \mathbb{R}^{d}$ with with $c_{t}=e_{t} w_{t}$ and $\int_{0}^{T}\left\|e_{t}\right\|_{L^{\infty}\left(B_{R}\right)} d t<+\infty$ for any $R>0$. Assume that b satisfies (5.1), (5.2), (5.3) and that there exist constants $C>0, R>0$ such that

$$
\left\|w_{t}^{i}\right\|_{\infty} \leqslant C, \quad w_{t}^{i}=0 \quad \mathcal{L}^{d} \text {-a.e. in } \mathbb{R}^{d} \backslash B_{R}
$$

for any $t \in(0, T)$. Then $w_{0}^{1} \leqslant w_{0}^{2}$ implies $w_{t}^{1} \leqslant w_{t}^{2}$ for any $t \in[0, T]$.

As showed in [21] and in the last section of [3], the comparison property can also be used as a tool to study the generic uniqueness of the ODE $\dot{\psi}=b(t, \psi)$, through the notion of regular Lagrangian flow. Let us recall the basic definitions in this context before stating the two relevant theorems in this discussion, concerning the existence and uniqueness for regular Lagrangian flows, and the stability of regular Lagrangian flows with respect to the approximation of $b$ with vector fields which are Lipshitz with respect to the $x$ variable.

Let $A \subset \mathbb{R}^{d}$ be a Borel set and let us denote by $\mathcal{S}\left(\mathbb{R}^{d}\right)$ the space $C\left([0, T] ; \mathbb{R}^{d}\right)$ endowed with the sup norm. Given an $\mathcal{L}^{d}$-measurable map 
$\psi: A \rightarrow \mathcal{S}\left(\mathbb{R}^{d}\right)$, we say that $\psi$ is a Lagrangian flow starting from $A$ relative to $b$ if $\psi(x)(\cdot)$ is an integral solution of the ODE $\dot{\gamma}=b(t, \gamma)$ starting at $x$ (i.e. $\gamma \in C\left([0, T] ; \mathbb{R}^{d}\right)$ and $\gamma(t)=x+\int_{0}^{t} b(\tau, \gamma(\tau)) d \tau$ for any $\left.t \in[0, T]\right)$ for $\mathcal{L}^{d}$-a.e. $x \in A$. Furthermore, we say that a Lagrangian flow $\psi$ is regular if there exist an increasing sequence of Borel sets $A_{h}$ whose union is $A$ and constants $C_{h}$ such that

$$
\left|\int_{A_{h}} \varphi(\psi(x)(t)) d x\right| \leqslant C_{h} \int_{\mathbb{R}^{d}}|\varphi(y)| d y \quad \forall \varphi \in C_{c}\left(\mathbb{R}^{d}\right), t \in[0, T] .
$$

The natural extension to the $S B D$ case of the results in Section VI of [3] are the following theorems:

Theorem 5.3 (EXISTEnCE AND UnIQUeness). - Assume that

(i) $b \in L^{\infty}\left((0, T) \times \mathbb{R}^{d} ; \mathbb{R}^{d}\right)$;

(ii) $b_{t} \in S B D_{\mathrm{loc}}\left(\mathbb{R}^{d}\right)$ for $\mathcal{L}^{1}$-a.e. $t \in(0, T),\left|E b_{t}\right|\left(B_{R}\right) \in L_{\mathrm{loc}}^{1}(0, T)$ for any $R>0$ and

$$
\int_{0}^{T}\left\|\left[\operatorname{div} b_{t}\right]^{-}\right\|_{L^{\infty}\left(B_{R}\right)} d t<+\infty \quad \forall R>0 .
$$

Then, for any $\mathcal{L}^{d}$-measurable $A \subset \mathbb{R}^{d}$ there exists a regular Lagrangian flow starting from $A$.

Furthermore, if $\psi_{i}$ are regular Lagrangian flows starting from $\mathcal{L}^{d}$-measurable set $A_{i} \subset \mathbb{R}^{d}, i=1,2$, we have

$$
\psi_{1}(x)=\psi_{2}(x) \quad \text { for } \mathcal{L}^{d} \text {-a.e. } x \in A_{1} \cap A_{2} .
$$

Theorem 5.4 (Stability). - Assume that (i) and (ii) in Theorem 5.3 hold. Let $b_{h}(t, x):(0, T) \times \mathbb{R}^{d} \rightarrow \mathbb{R}^{d}$ be satisfying

$$
\begin{gathered}
b_{h}(\cdot, x) \quad \text { is } \mathcal{L}^{1} \text {-measurable in }(0, T) \text { for any } x \in \mathbb{R}^{d}, \\
\sup _{h}\left\|b_{h}\right\|_{\infty} \leqslant C \quad \text { and } \quad b_{h} \rightarrow b \quad \text { in } L_{\text {loc }}^{1}\left((0, T) \times \mathbb{R}^{d} ; \mathbb{R}^{d}\right), \\
\nabla b_{h}(t, \cdot) \in L^{\infty}\left((0, T) \times B_{R} ; \mathbb{R}^{d \times d}\right) \quad \forall h \in \mathbb{N}, \forall R>0 .
\end{gathered}
$$

Let $\psi_{h}(x)(t)$ be the unique solutions in $[0, T]$ of the ODE $\dot{\gamma}(t)=b_{h}(t, \gamma(t))$ with the initial condition $\gamma(0)=x$ and assume that for any $R>0$ there exists a constant $C_{R}$ such that

$$
\left|\int_{B_{R}} \varphi\left(\psi_{h}(x)(t)\right) d x\right| \leqslant C_{R} \int_{B_{M_{R}}}|\varphi(y)| d y \quad \forall \varphi \in C_{c}\left(\mathbb{R}^{d}\right), t \in[0, T]
$$


with $M_{R}=R+T \sup _{h}\left\|b_{h}\right\|_{\infty}$. Then, denoting by $\psi$ the regular Lagrangian flow relative to $b$ starting from $\mathbb{R}^{d}$ given by Theorem 5.3, the functions $\psi_{h}$ converge in $L_{\text {loc }}^{1}\left(\mathbb{R}^{d} ; \mathcal{S}\left(\mathbb{R}^{d}\right)\right)$ to the function $\psi$, i.e.

$$
\lim _{h \rightarrow \infty} \int_{B_{R}[0, T]} \sup _{[0,}\left|\psi(x)(\cdot)-\psi_{h}(x)(\cdot)\right| d x=0 \quad \forall R>0 .
$$

\section{Continuity points, jump points and traces}

In this section we compare the trace operator, defined in the sense of distributions in Section 3, with the (approximate) pointwise limits defined with integral averages on balls, used in the definitions of the approximate discontinuity set $S_{u}$, the approximate limit $\tilde{u}$, the approximate jump set $J_{u}$ and the approximate one-sided limits $u^{ \pm}$. When $u=B \in B D_{\text {loc }}$ the following well known result provides a complete solution of this problem (see Chapter II in [28]):

Proposition 6.1. - Let $\Sigma \subset \Omega$ be a $C^{1}$ oriented hypersurface and $B \in B D_{\text {loc }}(\Omega)$. Then, up to $\mathcal{H}^{d-1}$-negligible sets, we have

$$
\operatorname{Tr}^{+}(B, \Sigma)= \begin{cases}\tilde{B} \cdot \nu_{\Sigma} & \text { on } \Sigma \backslash S_{B} \\ B^{+} \cdot \nu_{\Sigma} & \text { on } \Sigma \cap J_{B} \cap\left\{\nu_{B}=\nu_{\Sigma}\right\} \\ B^{-} \cdot \nu_{\Sigma} & \text { on } \Sigma \cap J_{B} \cap\left\{\nu_{B}=-\nu_{\Sigma}\right\}\end{cases}
$$

In addition, we have $\mathcal{H}^{d-1}\left(\left(S_{B} \backslash J_{B}\right) \cap \Sigma\right)=0$.

An analogous result holds for functions $u \in B V_{\text {loc }}\left(\Omega ; \mathbb{R}^{m}\right)$, see for instance Theorem 3.77 and Theorem 3.84 of [8]. In the following theorem we try to extend these properties to locally bounded scalar functions $w$ satisfying $w B \in \mathcal{M}_{\infty}(\Omega)$. It turns out that this is possible out of the set where the normal trace of $B$ on $\Sigma$ vanishes.

THEOREM 6.2 (ONE-SIDED LIMITS OF $w$ ON $C^{1}$ HYPERSURFACES). Let $\Sigma \subset \Omega$ be a $C^{1}$ oriented hypersurface and let $w, B$ be locally bounded functions with $B \in B D_{\text {loc }}(\Omega)$ and $w B \in \mathcal{M}_{\infty}(\Omega)$. Then, for $\mathcal{H}^{d-1}$-a.e. $x \in \Sigma$ such that $\operatorname{Tr}^{+}(B, \Sigma)(x) \neq 0$, we have (recall the notation (2.2))

$$
\lim _{r \downarrow 0} r^{-d} \int_{B_{r}^{+}\left(x, \nu_{\Sigma}(x)\right)}\left|w(y)-\frac{\operatorname{Tr}^{+}(w B, \Sigma)(x)}{\operatorname{Tr}^{+}(B, \Sigma)(x)}\right| d y=0 .
$$

An analogous result holds replacing $B_{r}^{+}\left(x, \nu_{\Sigma}(x)\right)$ with $B_{r}^{-}\left(x, \nu_{\Sigma}(x)\right)$ and $\operatorname{Tr}^{+}$with $\operatorname{Tr}^{-}$. 
Proof. - By the same extension argument used in the proof of Theorem 4.2 (based on Gagliardo's theorem on the surjectivity of the trace operator from $W^{1,1}$ to $L^{1}$ ) we can modify $w$ and $B$ only on the "negative" side of $\Sigma$ in order to obtain that $D \cdot(w B)\llcorner\Sigma=0$. This modification of $w$ and $B$ does not affect the statement of the theorem, due to the fact that the Lebesgue measure of the negative side of $\Sigma$ inside $B_{r}^{+}\left(x, \nu_{\Sigma}(x)\right)$ is an infinitesimal faster than $r^{d}$. Moreover, taking into account the change of variables for traces, the same extension ensures also that $D \cdot\left(w^{2} B\right)\llcorner\Sigma=0$.

Let $F \subset \Sigma$ be the set of points where $\operatorname{Tr}^{+}(B, \Sigma)=0$. We fix $x \in \Sigma \backslash F$ where the following properties hold:

(a) $x$ is a Lebesgue point for the maps $\operatorname{Tr}^{+}(w B, \Sigma)$ and $\operatorname{Tr}^{+}\left(w^{2} B, \Sigma\right)$, i.e.

$$
\lim _{r \downarrow 0} r^{1-d} \int_{B_{r}(x) \cap \Sigma}\left|\operatorname{Tr}^{+}(w B, \Sigma)(y)-\operatorname{Tr}^{+}(w B, \Sigma)(x)\right| d \mathcal{H}^{d-1}(y)=0
$$

and

$$
\lim _{r \downarrow 0} r^{1-d} \int_{B_{r}(x) \cap \Sigma}\left|\operatorname{Tr}^{+}\left(w^{2} B, \Sigma\right)(y)-\operatorname{Tr}^{+}\left(w^{2} B, \Sigma\right)(x)\right| d \mathcal{H}^{d-1}(y)=0 ;
$$

(b) $\operatorname{Tr}^{+}(B, \Sigma)(x) \operatorname{Tr}^{+}\left(w^{2} B, \Sigma\right)(x)=\left[\operatorname{Tr}^{+}(w B, \Sigma)(x)\right]^{2}$;

(c) $|D \cdot(w B)|\left(B_{r}(x)\right)=o\left(r^{d-1}\right)$ and $\left|D \cdot\left(w^{2} B\right)\right|\left(B_{r}(x)\right)=o\left(r^{d-1}\right)$;

(d) For some vector $\bar{B} \in \mathbb{R}^{d}$ we have

$$
\lim _{r \downarrow 0} r^{-d} \int_{B_{r}^{+}\left(x, \nu_{\Sigma}(x)\right)}|B(y)-\bar{B}| d y=0
$$

and $\bar{B} \cdot \nu_{\Sigma}(x)=\operatorname{Tr}^{+}(B, \Sigma)(x)$.

All the properties above hold out of an $\mathcal{H}^{d-1}$-negligible subset of $\Sigma \backslash F$ (recall the change of variables for traces, property (2.1) with $k=d-1$ and Proposition 6.1), hence if we show that these properties imply (6.1) we have completed our proof.

Up to a translation and a rotation we can assume with no loss of generality that $x=0$ and that $\nu_{\Sigma}(x)=e_{d}$, the $d$-th coordinate vector of the canonical basis of $\mathbb{R}^{d}$. Since $0 \notin F$ we have that $\bar{B}_{d} \neq 0$ and we can assume, to fix the ideas, that $\bar{B}_{d}>0$ (possibly replacing $B$ with $-B$ this is not restrictive). We describe $\Sigma$ in a neighbourhood of 0 as the 0 level set of a $C^{1}$ function $\Phi$, with $\nabla \Phi$ parallel to $\nu_{\Sigma}$, so that $\Phi(0)=0$ and $\nabla \Phi(0) /|\nabla \Phi(0)|=e_{d}$. 
Denoting by $x=\left(x^{\prime}, x_{d}\right)$ the generic point in $\mathbb{R}^{d}$, we consider the "parallelograms"

$$
Q_{r}:=\left\{\left(x^{\prime}, 0\right)+t \bar{B}: t \in\left(-\frac{r}{\bar{B}_{d}}, \frac{r}{\bar{B}_{d}}\right),\left\|x^{\prime}\right\|<r / 2\right\}
$$

(where $\left\|x^{\prime}\right\|=\sup \left\{\left|x_{i}\right|: 1 \leqslant i \leqslant d-1\right\}$ ) and their intersections $Q_{r}^{+}$with $\{\Phi>0\}$. Notice that the normal component of $\bar{B}$ vanishes on all faces of $Q_{r}$, with the exception of the "top" face $U_{r}$ (in the hyperplane $\left\{x_{d}=r\right\}$ ) and the "bottom" face $D_{r}$ (in the hyperplane $\left\{x_{d}=-r\right\}$ ). We denote by $\partial_{l} Q_{r}$ the boundary of $Q_{r}$ without the top and bottom faces. Notice also that $\mathcal{L}^{d}\left(Q_{r}^{+}\right)=r^{d}+o\left(r^{d}\right)$, since $\Sigma$ is a $C^{1}$ hypersurface.

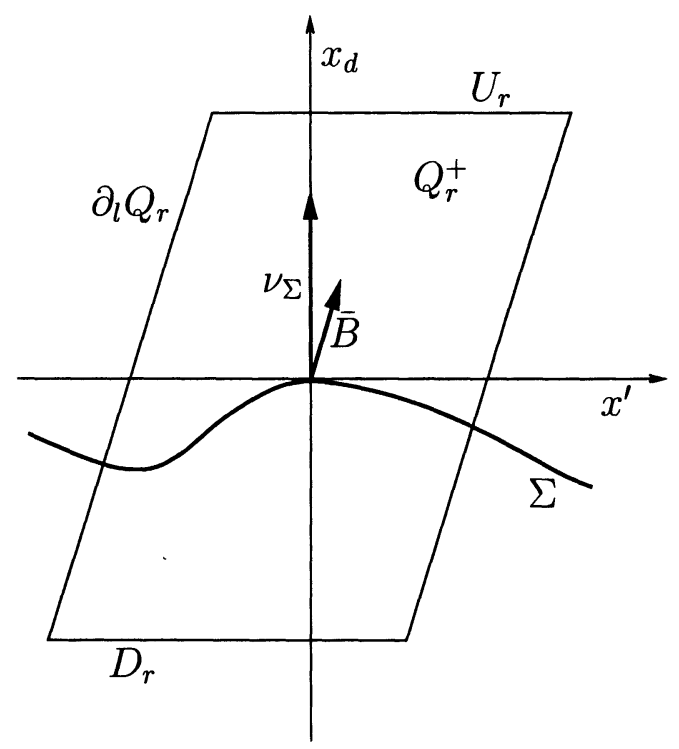

Let $r_{i} \downarrow 0$ and let $s_{i} \in\left(r_{i}, 2 r_{i}\right)$ be such that

$$
\int_{\partial Q_{s_{i}}}|B-\bar{B}| d \mathcal{H}^{d-1} \leqslant r_{i}^{-1} \int_{Q_{2 r_{2}}}|B-\bar{B}| d y
$$

and $\operatorname{Tr}^{+}\left(B, \partial Q_{s_{\imath}}\right)$ coincides $\mathcal{H}^{d-1}$-a.e. on $\partial Q_{s_{\imath}}$ with $B \cdot \nu, \nu$ being the outer normal to $Q_{s_{2}}$ (the existence of $s_{i}$ is ensured by the mean value theorem and by Proposition 3.6).

Step 1. We show first that

$$
\lim _{i \rightarrow \infty} s_{i}^{-d} \int_{Q_{s_{2}}^{+}} w B_{d} d y=\operatorname{Tr}^{+}(w B, \Sigma)(0) .
$$


Choose indeed the test function $\varphi_{i}(y)=s_{i}-y_{d}$ and apply the definition of trace to obtain

$-\int_{Q_{s_{\imath}}^{+}} w B_{d} d y=-\int_{Q_{s_{2}}^{+}} \varphi_{i} d(D \cdot(w B))+\int_{\partial Q_{s_{\imath}}^{+} \backslash U_{s_{\imath}}} \varphi_{i} \operatorname{Tr}\left(w B, \partial Q_{s_{i}}^{+}\right) d \mathcal{H}^{d-1}$,

because $\varphi_{i}=0$ on $U_{s_{i}}$. The first integral in the right hand side is $o\left(s_{i}^{d}\right)$, by condition (c) on the divergence of $w B$. The domain of integration in the second integral can be split into two parts, intersecting with $\partial Q_{s_{\imath}}$ (and this intersection is contained in $\partial_{l} Q_{s_{i}}$, for $i$ sufficiently large) and with $\Sigma$. Using the fact that $\bar{B} \cdot \nu=0$ on $\partial_{l} Q_{s_{\imath}}$, the first of these two parts is again $o\left(s_{i}^{d}\right)$, by the estimate

$$
\begin{aligned}
\int_{\partial_{l} Q_{s_{\imath}}}\left|\varphi_{i} \operatorname{Tr}\left(w B, \partial Q_{s_{2}}^{+}\right)\right| d \mathcal{H}^{d-1} & =\int_{\partial_{l} Q_{s_{2}}}\left|\varphi_{i}\right||w B \cdot \nu-w \bar{B} \cdot \nu| d \mathcal{H}^{d-1}(y) \\
& \leqslant \frac{2 s_{i}\|w\|_{L^{\infty}\left(Q_{1}\right)}}{r_{i}} \int_{Q_{2 r_{\imath}}}|B-\bar{B}| d y
\end{aligned}
$$

and by our choice of $s_{i}$. It remains to consider

$$
\int_{\partial Q_{s_{\imath}}^{+} \cap \Sigma} \varphi_{i} \operatorname{Tr}\left(w B, \partial Q_{s_{\imath}}^{+}\right) d \mathcal{H}^{d-1}=-\int_{\partial Q_{s_{\imath}}^{+} \cap \Sigma} \varphi_{i} \operatorname{Tr}^{+}(w B, \Sigma) d \mathcal{H}^{d-1} .
$$

Here we use condition (a) (that, by a comparison argument, holds for the family $Q_{r}$ as well) and the fact that $\varphi_{i}=s_{i}+o\left(s_{i}\right)$ on $Q_{s_{\imath}} \cap \Sigma$ to obtain that this integral is equal to

$$
-\operatorname{Tr}^{+}(w B, \Sigma)(0) s_{i}^{d}+o\left(s_{i}^{d}\right) .
$$

Step 2. In this step we show that

$$
\lim _{i \rightarrow \infty} s_{i}^{-d} \int_{Q_{s_{\imath}}^{+}}\left|w-\frac{\operatorname{Tr}^{+}(w B, \Sigma)(0)}{\operatorname{Tr}^{+}(B, \Sigma)(0)}\right| d y=0 .
$$

Indeed, repeating the argument of Step 1 for the vector field $w^{2} B$ (recall that properties (a) and (c) have been imposed for the vector field $w^{2} B$ as well) and using conditions (b) and (d) we obtain

$$
\lim _{i \rightarrow \infty} s_{i}^{-d} \int_{Q_{s_{\imath}}^{+}} w^{2} B_{d} d y=\operatorname{Tr}^{+}\left(w^{2} B, \Sigma\right)(0)=\frac{\left[\operatorname{Tr}^{+}(w B, \Sigma)(0)\right]^{2}}{\bar{B}_{d}}
$$

therefore condition (d) again gives

$$
\lim _{i \rightarrow \infty} s_{i}^{-d} \int_{Q_{s_{\imath}}^{+}} w^{2} B_{d}^{2} d y=\left[\operatorname{Tr}^{+}(w B, \Sigma)(0)\right]^{2} .
$$


Traces and fine properties of a $B D$ class of vector fields and applications

Expanding the squares and using (6.2) and (6.4) we get

$$
\lim _{i \rightarrow \infty} s_{i}^{-d} \int_{Q_{s_{i}}^{+}}\left|w B_{d}-\operatorname{Tr}^{+}(w B, \Sigma)(0)\right|^{2} d y=0 .
$$

Eventually, adding and subtracting $w \bar{B}_{d}$ and using condition (d) again we get (6.3).

Step 3. Conclusion. If $\bar{r}>0$ is such that $B_{\bar{r}}^{+}\left(0, e_{d}\right) \subset Q_{1}$, we obtain by Step 2 and by a comparison argument based on the fact that $B_{\bar{r} r}^{+}\left(0, e_{d}\right) \subset Q_{r}$ and that

$$
\mathcal{L}^{d}\left(B_{r}^{+}\left(0, e_{d}\right) \cap Q_{r} \backslash Q_{r}^{+}\right)=o\left(r^{d}\right)
$$

the property

$$
\lim _{i \rightarrow \infty} s_{i}^{-d} \int_{B_{\bar{r}_{s}}^{+}\left(0, e_{d}\right)}\left|w-\frac{\operatorname{Tr}^{+}(w B, \Sigma)(0)}{\operatorname{Tr}^{+}(B, \Sigma)(0)}\right| d y=0
$$

and therefore

$$
\lim _{i \rightarrow \infty} r_{i}^{-d} \int_{B_{\bar{r} r_{i}}^{+}\left(0, e_{d}\right)}\left|w-\frac{\operatorname{Tr}^{+}(w B, \Sigma)(0)}{\operatorname{Tr}^{+}(B, \Sigma)(0)}\right| d y=0 .
$$

Since the initial sequence $r_{i}$ is arbitrary the proof is achieved.

Remark 6.3 (One-sided approximate limits of $w$ on countably $\mathcal{H}^{d-1}$ rectifiable sets). - The previous result trivially extends to countably $\mathcal{H}^{d-1}$ rectifiable sets $\Sigma \subset \Omega$, where in this case $\nu_{\Sigma}$ is an orientation of $\Sigma$. Indeed, by the very definition of orientation, we can find countably many $C^{1}$ hypersurfaces $\Sigma_{i}$ and pairwise disjoint Borel sets $E_{i} \subset \Sigma_{i}$ such that $\mathcal{H}^{d}\left(\Sigma \backslash \cup_{i} E_{i}\right)=0$ and $\nu_{\Sigma}(x)$ is the classical normal to $\Sigma_{i}$ for any $x \in E_{i}$.

When the vector field has bounded variation we can say something more, proving existence of one-sided approximate limits $|D B|$-a.e. out of the singular sets where the normal component of $B$ vanishes. According to Alberti's rank one theorem [1] we can write $D^{s} B=\eta \otimes \xi\left|D^{s} B\right|$ for suitable unit vectors $\xi, \eta \in \mathbb{R}^{d}$ uniquely determined $\left|D^{s} B\right|$-a.e. up to a common change of sign. Comparing this representation with the one given in (2.4) on $J_{B}$, we see that we can orient $J_{B}$ in such a way that $\nu_{B}=\xi$. Then we define

$$
F_{B}^{+}:=\left\{x \in \Omega \backslash S_{B}: \tilde{B}(x) \cdot \xi(x) \neq 0\right\} \cup\left\{x \in J_{B}: B^{+}(x) \cdot \xi(x) \neq 0\right\},
$$




$$
F_{B}^{-}:=\left\{x \in \Omega \backslash S_{B}: \tilde{B}(x) \cdot \xi(x) \neq 0\right\} \cup\left\{x \in J_{B}: B^{-}(x) \cdot \xi(x) \neq 0\right\} .
$$

Observe that the sets $F_{B}^{ \pm}$depend on the choice of $\xi$, since $\xi$ is defined only $\left|D^{s} B\right|$-a.e. by Alberti's theorem. Nevertheless this ambiguity will not influence our results, because in Theorem 6.5 we will prove a property that holds $\left|D^{s} B\right|$-a.e. Furthermore notice that, once we choose $\xi, F_{B}^{+}$and $F_{B}^{-}$ differ only on $J_{B}$, where $B^{+}$and $B^{-}$could have different scalar products with $\xi$.

When $u=\chi_{E}$ is the characteristic function of a measurable set, the approximate discontinuity set $S_{u}$ coincides with the essential boundary $\partial^{*} E$, i.e. the set of points where the density is neither 0 nor 1 . On the other hand, choosing $\nu_{u}$ in such a way that $u^{+}>u^{-}$, it turns out that $u^{+}=1$ and $u^{-}=0$ at any approximate jump point. With this convention $\nu_{u}$ is called approximate inner normal to $E$ and we set $\nu_{E}=\nu_{u}$. When $u \in B V_{\mathrm{loc}}(\Omega)$ (i.e. the set $E$ has locally finite perimeter in $\Omega$ ) using a result due to De Giorgi and Federer (see Theorem 3.59 and Theorem 3.61 of [8]) we have

$$
\left|D \chi_{E}\right|=\mathcal{H}^{d-1}\left\llcorner\partial^{*} E \quad \text { and } \quad D \chi_{E}=\nu_{E} \mathcal{H}^{d-1}\left\llcorner\partial^{*} E\right. \text {. }\right.
$$

Lemma 6.4. - Let $B \in B V_{\text {loc }}\left(\Omega ; \mathbb{R}^{d}\right)$, let $i \in\{1, \ldots, d\}$ and let $E \subset \Omega$ be an $\mathcal{L}^{d}$-negligible Borel set. Then, for $\mathcal{L}^{1}$-a.e. $t \in \mathbb{R}$ the following property holds: $\left(\operatorname{sgn} \eta_{i}\right) \xi$ is the approximate inner normal to $\left\{B_{i}>t\right\}$ for $\mathcal{H}^{d-1}$-a.e. $x \in E \cap \partial^{*}\left\{B_{i}>t\right\}$.

Recall that $\operatorname{sgn} \eta_{i}$ is not defined only on a $\left|D^{s} B_{i}\right|$-negligible set, so the lemma is correctly stated, again in view of Theorem 6.5 .

Proof. - Without loss of generality we assume that $E \subset \subset \Omega$. We use (see for instance Theorem 3.40 of [8]) the coarea formulas and (6.7) to obtain for any Borel bounded map $\varphi$ with compact support in $\Omega$

$$
\begin{aligned}
& \int_{\Omega} \varphi d\left|D B_{i}\right|=\int_{\mathbb{R}} \int_{\partial^{*}\left\{B_{\imath}>t\right\}} \varphi d \mathcal{H}^{d-1} d t, \\
& \int_{\Omega} \varphi d D B_{i}=\int_{\mathbb{R}} \int_{\partial^{*}\left\{B_{\imath}>t\right\}} \varphi \xi_{t} d \mathcal{H}^{d-1} d t,
\end{aligned}
$$

where $\xi_{t}$ is the approximate inner normal to $\left\{B_{i}>t\right\}$. Using these formulas we get

$$
\begin{aligned}
\int_{\mathbb{R}} \mathcal{H}^{d-1}\left(E \cap \partial^{*}\left\{B_{i}>t\right\}\right) d t= & \left|D B_{i}\right|(E)=\left(\operatorname{sgn} \eta_{i}\right) \xi D B_{i}(E) \\
= & \int_{\mathbb{R}} \int_{E \cap \partial^{*}\left\{B_{\imath}>t\right\}}\left(\operatorname{sgn} \eta_{i}\right) \xi \cdot \xi_{t} d \mathcal{H}^{d-1} d t . \\
& -558-
\end{aligned}
$$


Comparing the two expressions we see that equality can hold only if the property stated in the lemma is true.

We can now prove existence of the approximate one-sided limits $|D B|$ a.e. out of the sets $F_{B}^{ \pm}$. By Lebesgue differentiation theorem it suffices to consider the singular part of $D B$ only.

THEOREM 6.5. - Let $B \in B V_{\mathrm{loc}}\left(\Omega ; \mathbb{R}^{d}\right)$. Then for $\left|D^{s} B\right|$-a.e. $x \in \Omega \backslash F_{B}^{+}$ we have

$$
\lim _{r \downarrow 0} r^{-d} \int_{B_{r}^{+}(x, \xi(x))}\left|w(y)-w^{+}(x)\right| d y=0
$$

for a suitable $w^{+}(x) \in \mathbb{R}$. Moreover, in any Borel set $E \subset \Omega \backslash\left(F_{B}^{+} \cup S_{B}\right)$ such that $\left|D^{s} B\right|\left\llcorner E \ll\left|D B_{i}\right|\right.$, we can characterize $w^{+}$by

$$
w^{+}(x)=\frac{\operatorname{Tr}^{+}\left(w B, \partial^{*}\left\{B_{i}>\tilde{B}_{i}(x)\right\}\right)(x)}{\operatorname{Tr}^{+}\left(B, \partial^{*}\left\{B_{i}>\tilde{B}_{i}(x)\right\}\right)(x)} \quad \text { for }\left|D^{s} B\right|-\text { a.e. } x \in E \text {. }
$$

Proof. - Since $S_{B}$ is countably $\mathcal{H}^{d-1}$-rectifiable the existence of the one-sided approximate limit on $S_{B}$ is a direct consequence of Theorem 6.2 and of Remark 6.3. Therefore, we consider in the following only points out of $F_{B}^{+} \cup S_{B}$.

Since $\left|D^{s} B\right| \ll \sum_{i}\left|D^{s} B_{i}\right|$ we can find pairwise disjoint and $\mathcal{L}^{d}$-negligible Borel sets $E_{1}, \ldots, E_{d}$ such that $\left|D^{s} B\right| L E_{i} \ll\left|D^{s} B_{i}\right|$ and the union of the $E_{i}$ 's covers $\left|D^{s} B\right|$-almost all of $\Omega \backslash\left(F_{B}^{+} \cup S_{B}\right)$. We will prove that $w^{+}$exists for $|D B|$-a.e. $x \in E_{i}$ and

$$
w^{+}(x)=\frac{\operatorname{Tr}^{+}\left(w B, \partial^{*}\left\{B_{i}>\tilde{B}_{i}(x)\right\}\right)(x)}{\operatorname{Tr}^{+}\left(B, \partial^{*}\left\{B_{i}>\tilde{B}_{i}(x)\right\}\right)(x)} \quad \text { for }\left|D^{s} B_{i}\right| \text {-a.e. } x \in E_{i} .
$$

By Lemma 6.4 we have that for $\mathcal{L}^{1}$-a.e. $t \in \mathbb{R}$ the following two properties hold: first, $\left\{B_{i}>t\right\}$ has locally finite perimeter in $\Omega$; second, the approximate unit normal to $\left\{B_{i}>t\right\}$ is given by $\left(\operatorname{sgn} \eta_{i}\right) \xi(x)$ for $\mathcal{H}^{d-1}$ a.e. $x \in E_{i} \cap \partial^{*}\left\{B_{i}>t\right\}$. For any such $t$, by Remark 6.3, Theorem 6.2 and by rectifiability of $\partial^{*}\left\{B_{i}>t\right\}$, we obtain that (6.8) holds for $\mathcal{H}^{d-1}$-a.e. $x \in E_{i} \cap \partial^{*}\left\{B_{i}>t\right\}$, with

$$
w^{+}(x)=\frac{\operatorname{Tr}^{+}\left(w B, \partial^{*}\left\{B_{i}>t\right\}\right)(x)}{\operatorname{Tr}^{+}\left(B, \partial^{*}\left\{B_{i}>t\right\}\right)(x)} .
$$

Since $E_{i}$ does not intersect $S_{B}$ we have that $t=\tilde{B}_{i}(x)$ for any $x \in E_{i} \cap$ $\partial^{*}\left\{B_{i}>t\right\}$. Indeed, if $x$ belongs to $\partial^{*}\left\{B_{i}>t\right\}$ and $t<\tilde{B}_{i}(x)$, then 
$\mathcal{L}^{d}\left(\left\{B_{i} \leqslant t\right\} \cap B_{r}(x)\right)$ should not be $o\left(r^{d}\right)$, but using Chebyshev inequality we should have

$$
\begin{aligned}
& \left(\tilde{B}_{i}(x)-t\right) \mathcal{L}^{d}\left(\left\{B_{i} \leqslant t\right\} \cap B_{r}(x)\right) \\
\leqslant & \left(\tilde{B}_{i}(x)-t\right) \mathcal{L}^{d}\left(\left\{\left|B_{i}-\tilde{B}_{i}(x)\right| \geqslant \tilde{B}_{i}(x)-t\right\} \cap B_{r}(x)\right) \\
\leqslant & \int_{B_{r}(x)}\left|B_{i}-\tilde{B}_{i}(x)\right|
\end{aligned}
$$

and this integral is $o\left(r^{d}\right)$ because $x \notin S_{B}$. In the same way we can prove that $t$ cannot be strictly greater than $\tilde{B}_{i}(x)$. Therefore the formula for $w^{+}(x)$ given in (6.9) holds and finally, by integration with respect to $t$, the coarea formula gives the existence of $w^{+}$for $\left|D^{s} B_{i}\right|$-a.e. $x \in E_{i}$.

\section{Bibliography}

[1] Alberti (G.). - Rank-one properties for derivatives of functions with bounded variation. Proc. Roy. Soc. Edinburgh Sect. A, 123, p. 239-274 (1993).

[2] Alberti (G.), Ambrosio (L.). - A geometrical approach to monotone functions in $\mathbb{R}^{n}$. Math. Z., 230, p. 259-316 (1999).

[3] Ambrosio (L.). - Transport equation and Cauchy problem for $B V$ vector fields. Invent. Math., 158, p. 227-260 (2004).

[4] Ambrosio (L.), Bouchut (F.), De Lellis (C.). - Well-posedness for a class of hyperbolic systems of conservation laws in several space dimensions. Comm. Partial Diff. Eq., 29, p. 1635-1651 (2004).

[5] Ambrosio (L.), Coscia (A.), Dal Maso (G.). - Fine properties of functions in $B D$. Arch. Rat. Mech. Anal., 139, p. 201-238 (1997).

[6] Ambrosio (L.), De Lellis (C.). - Existence of solutions for a class of hyperbolic systems of conservation laws in several space dimensions. IMRN, 41, p. 2205-2220 (2003).

[7] Ambrosio (L.), De Lellis (C.), Maly (J.). - On the chain rule for the divergence of $B V$ like vector fields: applications, partial results, open problems. Preprint (2005).

[8] Ambrosio (L.), Fusco (N.), Pallara (D.). - Functions of bounded variation and free discontinuity problems. Oxford Mathematical Monographs (2000).

[9] Anzellotti (G.). - Pairings between measures and bounded functions and compensated compactness. Ann. Mat. Pura App., 135, p. 293-318 (1983).

[10] Anzellotti (G.). - The Euler equation for functionals with linear growth. Trans. Amer. Mat. Soc., 290, p. 483-501 (1985).

[11] Anzellotti (G.). - Traces of bounded vectorfields and the divergence theorem. Unpublished preprint (1983).

[12] Bouchut (F.). - Renormalized solutions to the Vlasov equation with coefficients of bounded variation. Arch. Rational Mech. Anal., 157, p. 75-90 (2001). 
Traces and fine properties of a $B D$ class of vector fields and applications

[13] Bouchut (F.), James (F.). - One dimensional transport equation with discontinuous coefficients. Nonlinear Analysis, 32, p. 891-933 (1998).

[14] Bouchut (F.), James (F.), Mancini (S.). - Uniqueness and weak stablity for multi-dimensional transport equations with one-sided Lipschitz coefficients. Ann. Scuola Normale Superiore di Pisa, Classe di Scienze, (5) 4, p. 1-25 (2005).

[15] Bressan (A.). - An ill posed Cauchy problem for a hyperbolic system in two space dimensions. Rend. Sem. Mat. Univ. Padova, 110, p. 103-117 (2003).

[16] Capuzzo Dolcetta (I.), Perthame (B.). - On some analogy between different approaches to first order PDE's with nonsmooth coefficients. Adv. Math. Sci Appl., 6, p. 689-703 (1996).

[17] Chen (G.-Q.), Frid (H.). - Divergence-measure fields and conservation laws. Arch. Rational Mech. Anal., 147, p. 89-118 (1999).

[18] Chen (G.-Q.), Frid (H.). - Extended divergence-measure fields and the Euler equation of gas dynamics. Comm. Math. Phys., 236, p. 251-280 (2003).

[19] Colombini (F.), Lerner (N.). - Uniqueness of continuous solutions for $B V$ vector fields. Duke Math. J., 111, p. 357-384 (2002).

[20] Colombini (F.), Lerner (N.). - Uniqueness of $L^{\infty}$ solutions for a class of conormal $B V$ vector fields. Geometric Analysis of PDE and Several Complex Variables, Contemp. Math., 368, p. 133-156 (2005).

[21] Di Perna (R.J.), Lions (P.L.). - Ordinary differential equations, transport theory and Sobolev spaces. Invent. Math., 98, p. 511-547 (1989).

[22] Evans (L.C.), GariePY (R.F.). - Lecture notes on measure theory and fine properties of functions, CRC Press (1992).

[23] Federer (H.). - Geometric measure theory, Springer (1969).

[24] Keyfitz (B.L.), Kranzer (H.C.). - A system of nonstrictly hyperbolic conservation laws arising in elasticity theory. Arch. Rational Mech. Anal., 72, p. 219-241 (1980).

[25] Lions (P.L.). - Sur les équations différentielles ordinaires et les équations de transport. C. R. Acad. Sci. Paris Sér. I, 326, p. 833-838 (1998).

[26] Petrova (G.), Popov (B.). - Linear transport equation with discontinuous coefficients. Comm. PDE, 24, p. 1849-1873 (1999).

[27] Popaud (F.), RAscle (M.). - Measure solutions to the liner multidimensional transport equation with non-smooth coefficients. Comm. PDE, 22, p. 337-358 (1997).

[28] Temam (R.). - Problèmes mathématiques en plasticité. Gauthier-Villars, Paris (1983).

[29] VASSEUR (A.). - Strong traces for solutions of multidimensional scalar conservation laws. Arch. Ration. Mech. Anal., 160, p. 181-193 (2001). 